\title{
Etiology of Branch Dieback and Shoot Blight of English Walnut Caused by Botryosphaeriaceae and Diaporthe Species in Southern Spain
}

\author{
Ana López-Moral, ${ }^{1}$ María Lovera, ${ }^{2}$ María del Carmen Raya, ${ }^{1}$ Nerea Cortés-Cosano, ${ }^{1}$ Octavio Arquero, ${ }^{2}$ Antonio Trapero, \\ and Carlos Agustí-Brisach ${ }^{1, \dagger}$ \\ ${ }^{1}$ Departamento de Agronomía, ETSIAM, Universidad de Córdoba, Campus de Rabanales, 14071 Córdoba, Spain \\ ${ }^{2}$ Departamento de Fruticultura Mediterránea, IFAPA, 14004 Córdoba, Spain
}

\begin{abstract}
English walnut (Juglans regia L.) is considered an economically important fruit crop worldwide. In Spain, little attention has been given to walnut diseases owing to the minor economic importance of the walnut crop in the country until recently. In 2017, typical symptoms of branch dieback and shoot blight of English walnut were observed in southern Spain. From 2017 to 2018, 10 commercial walnut orchards showing disease symptoms were surveyed. Botryosphaeriaceae and Diaporthe fungi were consistently isolated from affected shoots. Cytospora isolates were also recovered with minor relevance. Representative isolates of each fungal group were characterized based on colony and conidial morphology, optimum growth temperature, and comparison of DNA sequence data from the internal transcribed spacer, elongation factor $1-\alpha$, and $\beta$-tubulin ge-

growth temperatures $\left(\approx 25\right.$ to $\left.27^{\circ} \mathrm{C}\right)$ than Cytospora sp. $\left(19.5^{\circ} \mathrm{C}\right)$. The following species were identified: Botryosphaeriaceae: Botryosphaeria dothidea, Diplodia seriata, Dothiorella sarmentorum, Dothiorella sp., Neofusicoccum mediterraneum, and N. parvum; Diaporthe: Diaporthe neotheicola, Dia. rhusicola, Diaporthe sp., and Phomopsis amygdali; and Cytospora sp. Botryosphaeriaceae isolates were the most aggressive fungi to walnut in all tissues evaluated, followed by Diaporthe isolates and Cytospora sp. $N$. parvum was the most virulent among the remaining species tested in any of the tissues evaluated, followed by $B$. dothidea or $N$. mediterraneum. This work is the first report to identify the fungal species causing this complex disease of English walnut in Spain and Europe.
\end{abstract} nomic areas. Pathogenicity tests were performed on detached and attached shoots and on detached fruit by inoculating them with mycelial plugs. Botryosphaeriaceae and Diaporthe isolates had higher optimum
Keywords: canker diseases, diagnosis, identification, Juglans regia, pathogenicity, tree nuts
English walnut (Juglans regia L.) is considered an economically important tree cultivated for nuts and wood production worldwide. China and the United States are the main producer countries of nuts, with $43 \%(880,000 \mathrm{t})$ and $30 \%(611,280 \mathrm{t})$ of the total fruit production in the world, respectively (International Nut and Dried Fruit Council 2017; López-Larrinaga et al. 2017). Nevertheless, despite the favorable edaphoclimatic and orographic conditions of the Iberian Peninsula's lands for this crop, to date the economic impact of the English walnut crop in Spain has been low (Lovera et al. 2017a, 2017b). Indeed, the total growing surface of this nut crop in Spain is only approximately 10,367 ha, with $15,744 \mathrm{t}$ of nut production. The main walnut growing region in this country is Andalusia (southern Spain), with approximately 2,218 ha of cultivated area and 2,538 t of nut production, followed by Extremadura (westsouthern Spain; 1,645 ha, 2,414 t) and Castilla La Mancha (central Spain; 1,473 ha, 1,305 t) (López-Larrinaga et al. 2017; Ministerio de Agricultura, Pesca y Alimentación 2017). In addition, the European Union has a high deficit in nut production, and its market depends markedly on international imports. Additional advantages of this nut crop, such as its high rentability and the high level of mechanization of the cultural practices available for walnut growers,

${ }^{\dagger}$ Corresponding author: C. Agustí-Brisach; cagusti@uco.es

Funding: This research was funded by the Junta de Andalucía (project 'PPTRATRA-2016.00.6. Transforma de Fruticultura Mediterránea' from the Andalusian Institute for Research and Formation in Agriculture and Fishery, IFAPA). C. Agustí-Brisach is the holder of a 'Juan de la CiervaIncorporación' fellowship funded by the Spanish Ministry of Science, Innovation and Universities (MICINN).

The author(s) declare no conflict of interest.

Accepted for publication 1 August 2019.

(C) 2020 The American Phytopathological Society implicate the English walnut as a potential alternative nut crop in Spain (Lovera et al. 2017a, 2017b). In fact, the Spanish walnut growing surface increased by $13 \%$ of the cultivated surface between 2013 and 2016 (López-Larrinaga et al. 2017).

In Spain, little attention has been given to research on walnut diseases owing to the minor economic importance of this nut crop in the country until recently. Likewise, fruit diseases are traditionally considered the most important disorders that cause economic yield losses in this crop. Studies conducted recently in southern Spain by Lovera et al. (2017a, 2017b) indicated that the bacterium Xanthomonas arboricola pv. juglandis is the main causal agent of walnut blight and is associated with many other bacteria and fungi that can coinfect the affected nuts, increasing the disease severity. Nevertheless, since late spring to summer 2017, typical symptoms of shoot blight including spur, twig, bud, and leaf blight and branch dieback with wood canker formation and internal wood discoloration have been observed in commercial English walnut orchards in southern Spain. Although the disease has been previously described in California, causing serious damage to the commercial English walnut orchards located in the main nut growing counties in San Joaquín and Sacramento Valleys (Chen et al. 2014; Michailides and Hasey 2010), the etiology and biology of the disease are still uncertain in other walnut growing regions of the world, such as Spain.

Previous studies revealed that Botryosphaeriaceae and Diaporthe (syn. Phomopsis) are the main fungal species associated with the symptoms described above in English walnut (Chen et al. 2014; Li et al. 2015; Michailides and Hasey 2010). It is well known that Botryosphaeriaceae and Diaporthe fungi are widespread worldwide, causing many important diseases of woody crops, including fruit rots, branch dieback, shoot blight, and stem cankers, among other symptoms (Guarnaccia et al. 2018; Phillips et al. 2008). To date, the following 12 fungal species belonging to these two groups of fungi have been described as causing branch dieback and shoot blight of English walnut in California: (i) Botryosphaeriaceae: Botryosphaeria dothidea, Diplodia mutila, D. seriata, Dothiorella iberica, Lasiodiplodia citricola, Neofusicoccum mediterraneum, N. nonquaesitum, 
N. parvum, N. vitifusiforme, and Neoscytalidium dimidiatum; (ii) Diaporthe: Diaporthe neotheicola (syn. Dia. foeniculacea, Dia. foeniculina) and Dia. rhusicola (Chen et al. 2014; Michailides and Hasey 2010; Trouillas et al. 2010). Additionally, B. dothidea and Lasiodiplodia pseudotheobromae have also been associated with the disease in China (Li et al. 2015) and D. mutila in Chile (Díaz et al. 2018). Juglanconis juglandina, belonging to Diaporthales, has been identified as a causal agent of cankers in J. nigra and J. regia. The asexual morph of this species is common in Asia and Europe (Voglmayr et al. 2017).

Species belonging to the genus Cytospora have also been described as destructive canker pathogens that cause dieback in a broad range of woody plant species, including Juglans and Prunus spp. (Lawrence et al. 2017). In China, Cytospora atrocirrhata, C. chrysosperma, C. gigalocus, $C$. nivea, and $C$. sacculus were identified as causal agents of branch dieback and cankers in English walnut (Fan et al. 2015; Zhao et al. 2018).

Finally, other canker diseases, such as thousand cankers disease, have also been described as affecting Juglans species in forests. Wilting, yellowing of leaves, and branch dieback are the typical symptoms of the disease. Thousand cankers disease is caused by the fungus Geosmithia morbida, which is widely distributed across the western United States, and it is vectored primarily by the walnut twig beetle Pityophthorus juglandis (Daniels et al. 2016).

With respect to canker diseases in Spain, many Botryosphaeriaceae species have been previously reported to be associated with branch dieback and general decline in the main woody crops growing in this country, such as almond (Gramaje et al. 2012; Olmo et al. 2017), grapevine (Agustí-Brisach et al. 2019a; Armengol et al. 2001; Aroca et al. 2006, 2008; Martin and Cobos 2007), and olive (Agustí-Brisach et al. 2017; Moral et al. 2010, 2017), as well as in forests, such as Quercus spp. (Sánchez et al. 2003). Concerning Diaporthe species, Phomopsis amygdali has been described as causing cankers and wood decay of almond in Spain (Gramaje et al. 2012; Olmo et al. 2017). Recently, Guarnaccia et al. (2018) conducted a broad survey of grapevines in Europe to understand the Diaporthe diversity affecting this crop. In particular, the following Diaporthe species have been associated with Phomopsis cane and leaf spot of grapevine in Spain: Dia. ambigua, Dia. ampelina, Dia. baccae, Dia. eres, Dia. hispaniae, Dia. hungariae, and Dia. rudis (Guarnaccia et al. 2018). In Spain, several Cytospora spp. have been described causing cankers in woody crops and forests (Melgarejo et al. 2010; Sánchez-Solana et al. 2018). Recently, Cytospora pruinosa has been reported for the first time in this country as causing branch dieback of olive with low severity (Moral et al. 2017). However, this genus has not been extensively studied in tree nuts in Spain.

Botryosphaeriaceae, Cytospora, and Diaporthe fungi are considered saprophytes and endophytes, and they are able to survive on dead or inactive plant tissues (i.e., bark or wood of trees). Many of those pathogenic species have opportunistic behavior, and they are considered facultative wound parasites that attack weakened hosts (Agustí-Brisach et al. 2019b; Gomes et al. 2013; Guarnaccia et al. 2018; Lawrence et al. 2017; Phillips et al. 2008; Úrbez-Torres 2011; Úrbez-Torres and Gubler 2009; Úrbez-Torres et al. 2006,
2013). These pathogens develop fruiting bodies (pycnidia) on dead tissues and produce viable spores over long periods of time, providing the spore source to infect plants through natural (i.e., fruit abscission point, leaf scars, etc.) or artificial wounds (i.e., pruning wounds) (Michailides and Hasey 2010; Moral et al. 2019; Rolshausen et al. 2010; Úrbez-Torres et al. 2013). In addition, coinfections between Botryosphaeriaceae and Diaporthe species could cause a possible retarding or antagonistic effect on infection and disease development. In fact, studies conducted in California by Agustí-Brisach et al. (2019b) showed a significant delay in lesion development in shoots and hulls of English walnut when Dia. rhusicola was inoculated 4 days before $N$. mediterraneum with respect to the other interaction treatments evaluated.

Although studies on the etiology and epidemiology of branch dieback and shoot blight of English walnut have been conducted recently in the two main growing regions of this crop in the world (United States and China), the etiology of the disease in Spain has not yet been studied. Moreover, the extension of this nut crop to growing areas with favorable environmental conditions for disease development, such as southern Spain, has contributed to a major incidence of this complex disease during the last few years. Studies on etiology are essential for a good disease diagnosis to develop future research on the epidemiology and control of the disease. Therefore, the objectives of this work were to (i) identify the different fungal species associated with branch dieback and shoot blight of English walnut by morphology and molecular phylogeny and (ii) evaluate their pathogenicity to walnut shoots and green fruit (epicarp) under laboratory and field conditions.

\section{Materials and Methods}

Field surveys and fungal isolation. From spring 2017 to summer 2018, field surveys were conducted to study the occurrence of branch dieback and shoot blight of English walnut in southern Spain. Ten commercial walnut orchards belonging to Andalusia (Almería, Granada, Málaga, and Sevilla provinces) and Extremadura regions (Badajoz Province) were surveyed. These fields grew the English walnut cultivars (cvs.) Chandler, Hartley, Serr, Tulare, or Vina, with ages ranging from 12 to 35 years (Table 1). In each orchard, at least three walnut trees showing symptoms of branch dieback and shoot blight were examined, and the affected branches and shoots were cut. Wood samples showing internal discoloration and/or pycnidia on the bark were collected, placed in plastic bags, and kept at $4^{\circ} \mathrm{C}$ until isolations were made in the laboratory.

Once in the laboratory, the outer bark of affected wood samples was removed. Subsequently, small wood fragments from the margin of the affected area of each symptomatic branch were surface disinfested by dipping the fragments into a commercial bleach $(\mathrm{Cl}$ at $50 \mathrm{~g} /$ liter) solution at $10 \%$ (vol/vol) in sterile water for $2 \mathrm{~min}$, air dried on sterile filter paper for $15 \mathrm{~min}$, and plated on potato dextrose agar (PDA; Difco Laboratories, Le Pont de Claix, France) acidified with lactic acid $(2.5 \mathrm{ml}$ of $25 \%$ [ $\mathrm{vol} / \mathrm{vol}]$ per liter of medium) (APDA). When fungal fruiting structures were present on the affected tissues, they were removed carefully with a sterile needle and plated directly

Table 1. Commercial walnut orchards showing branch dieback and shoot blight surveyed between 2017 and 2018 in southern Spain

\begin{tabular}{llccc}
\hline Field & Location (municipality/province/region) & Walnut cultivar & Age of trees (years) & Date of survey (month/day/year) \\
\hline 1 & Antequera, Málaga, Andalucía & Vina & 12 & $04 / 11 / 2017$ \\
2 & Benavides, Badajoz, Extremadura & Tulare & 15 & $07 / 14 / 2017$ \\
3 & Benavides, Badajoz, Extremadura & Serr & 15 & $07 / 14 / 2017$ \\
4 & Badajoz, Badajoz, Extremadura & Chandler & 35 & $07 / 10 / 2017$ \\
5 & Guadix, Granada, Andalucía & Chandler & 14 & $07 / 26 / 2017$ \\
& & & & $07 / 19 / 2018$ \\
6 & Chirivel, Almería, Andalucía & Chandler & 15 & $07 / 27 / 2017$ \\
7 & Alcalá del Río, Sevilla, Andalucía & Chandler & 16 & $10 / 18 / 2017$ \\
8 & Badajoz, Badajoz, Extremadura & Hartley & 35 & $11 / 03 / 2017$ \\
9 & Badajoz, Badajoz, Extremadura & Serr & 30 & $11 / 03 / 2017$ \\
10 & Potted plants of commercial nursery & Vina & 2 & $07 / 12 / 2018$ \\
\hline
\end{tabular}


onto APDA. A total of 630 wood pieces obtained from the margin of the affected tissues or fruiting bodies were plated on Petri dishes for fungal isolation (inoculation points on Petri dishes: 10 orchards $\times 3$ walnut trees $\times 3$ Petri dishes $\times 7$ wood pieces or fruiting bodies per Petri dish). Petri dishes were incubated for 5 to 14 days at $25^{\circ} \mathrm{C}$ in darkness until colonies were large enough to be examined. To obtain pure cultures, hyphal tips from the margin of the fungal colonies were transferred to PDA and incubated as described above. Based on our previous experience in the diagnosis of fungal trunk pathogens, and according to colony color and mycelial growth development, fungal isolates from each orchard were grouped into three fungal groups: Botryosphaeriaceae (light to dark gray, fast-growing mycelium), Diaporthe (beige-white, middle- to fast-growing mycelium) or Cytospora (beige to olive gray, middle- to slow-growing mycelium). Pycnidia production was induced when it was necessary to observe conidia (see the protocol below). These morphological observations were helpful in selecting 39 representative isolates that were used for further analysis (Table 2). To obtain pure cultures, all the isolates were single-spored prior to morphological and molecular identification using the serial dilution method described by Dhingra and Sinclair (1995). They were transferred to sterile plastic tubes filled with PDA, and sterile paraffin oil (Panreac Química SA, Barcelona, Spain) was added after colonization for long-term storage. The studied isolates are registered and maintained at $4{ }^{\circ} \mathrm{C}$ in darkness in the collection of the Department of Agronomy at the University of Cordoba, Spain.

Fungal colony, conidia, and pycnidia morphology. Based on colony characteristics (texture, density, color, zonation, margin, and growth rate), isolates collected from walnut trees showing branch dieback were first identified at the family or genus level. According to this identification, 10 representative isolates (Table 2) of the different types of colonies observed were used to study colony morphology after growing the isolates on PDA at $25 \pm 2{ }^{\circ} \mathrm{C}$ in darkness. Botryosphaeriaceae, Cytospora, and Diaporthe fungi were first identified at the genus level by colony morphology when grown on PDA (Gomes et al. 2013; Phillips et al. 2013; Norphanphoun et al. 2017). To induce pycnidia production for conidium and pycnidium characterization, all fungal isolates were grown on pistachio (Pistacia vera L.) leaf agar (LA) (Agustí-Brisach et al. 2019b; Chen et al. 2014) at $25^{\circ} \mathrm{C}$ for 3 weeks under continuous fluorescent light.

Characteristics of mycelia were recorded after 4 to 10 days (depending on the mycelial growth development of each isolate) of incubation at $25^{\circ} \mathrm{C}$ in darkness (Barnett and Hunter 1998), and color was determined with a color scale (Kornerup and Wanscher 1963). For conidial measurements, conidial masses from pycnidia obtained from the surface of LA were placed on slides with a drop of $0.01 \%$ acid fuchsine in lactoglycerol (1:2:1 lactic acid/glycerol/water) and covered with a coverslip. Fungal structures were observed and measured at a $\times 400$ magnification or at a $\times 1,000$ magnification under the oil-immersion lens, depending on the conidial size, using a Nikon Eclipse 80i microscope (Nikon, Tokyo, Japan). Pycnidia were measured directly from the surface of LA at a $\times 20-7.5$ magnification using a Nikon SMZ18 binocular. For each isolate, 30 measurements were obtained for each structure. The average length and width were calculated. The length/width ratio was also obtained for conidia.

Effect of temperature on mycelial growth. The 10 representative isolates selected in this study for morphological characterization (Table 2) were grown on PDA at $25^{\circ} \mathrm{C}$ in darkness for 10 days. Mycelial plugs (7.5-mm diameter) obtained from the margins of actively growing colonies were placed in the center of Petri dishes filled with PDA. Petri dishes were incubated at 5, 10, 15, 20, 25, 30, and $35^{\circ} \mathrm{C}$ in darkness. The largest and smallest diameters of the colonies were measured with a digital scale ruler from 4 to 10 days after inoculation, depending on the mycelial growth development of each isolate. Mean data were converted to radial growth rate $(\mathrm{mm} / \mathrm{day})$. There were four replicate Petri dishes per isolate and temperature combination, and the experiment was conducted twice.

For each isolate, a nonlinear adjustment of the data was applied using the generalized Analytics Beta model (Hau and Kranz 1990) to evaluate the variation in mycelial growth rate over temperature
(López-Moral et al. 2017). For each isolate, the average growth rates at each temperature were adjusted to a regression curve to estimate the optimum growth temperature and the maximum growth rate (MGR). Data were tested for normality and homogeneity of variances, and ANOVA was performed with the optimum growth temperature or the MGR as dependent variables and isolate as the independent variable. For each variable, isolate means were compared according to Tukey's honestly significant difference (HSD) test at $\alpha=0.05$ (Steel and Torrie 1985). Data from this experiment were analyzed using Statistix 10 software (Analytical Software 2013).

Molecular characterization. DNA extraction. Genomic DNA was obtained from the active mycelium of the 39 fungal isolates belonging to the Botryosphaeriaceae, Cytospora, and Diaporthe fungi included in this study (Table 2) that were growing on PDA as described above. Mycelial tissues were ground using the FastPrep-24 grinder machine (MP Biomedicals, Santa Ana, CA). Subsequently, DNA extractions were performed using the E.Z.N.A. Fungal DNA Kit (OMEGA BioTek, Norcross, GA) following the manufacturer's instructions. A MaestroNano spectrophotometer (MaestroGen, Taiwan) was used to determine the concentration and purity of the extracted DNA.

PCR analysis and sequencing. For all of the isolates, the 5.8S nuclear ribosomal gene with two flanking internal transcribed spacers (ITS) and the genomic areas elongation factor $1-\alpha(\mathrm{EF})$ and $\beta$-tubulin (TUB) were amplified. ITS was amplified using the primer pair ITS4/ ITS5 (White et al. 1990), and EF and TUB were amplified with the primer pairs EF1-728F/EF1-986R (Carbone and Kohn 1999) and T1 (O'Donnell and Cigelnik 1997)/Bt2b (Glass and Donaldson 1995), respectively. The PCRs were performed in a total volume of $25 \mu \mathrm{l}$ containing the following mixture: $20 \mathrm{ng}$ of genomic DNA, $5 \mu \mathrm{l}$ of $5 \times$ My Taq Reaction Buffer, and $0.13 \mu$ l of My Taq DNA Polymerase (Bioline, Memphis, TN). Additionally, $0.2 \mu \mathrm{M}$ (each) primer was used for the EF and TUB PCRs, whereas for the ITS region PCR, $0.4 \mu \mathrm{M}$ (each) primer was added. A negative control was included in all PCRs using ultrapure water instead of DNA. The PCR cycling programs were conducted as follows: for ITS, initial denaturation at $94^{\circ} \mathrm{C}$ for $3 \mathrm{~min}$, followed by 30 cycles of $94^{\circ} \mathrm{C}$ for $30 \mathrm{~s}, 48^{\circ} \mathrm{C}$ for 30 $\mathrm{s}$, and $72^{\circ} \mathrm{C}$ for $1 \mathrm{~min}$, and a final extension at $72^{\circ} \mathrm{C}$ for $10 \mathrm{~min}$; for $\mathrm{EF}$, initial denaturation at $94^{\circ} \mathrm{C}$ for $3 \mathrm{~min}$, followed by 35 cycles of $94^{\circ} \mathrm{C}$ for $30 \mathrm{~s}, 50^{\circ} \mathrm{C}$ for $30 \mathrm{~s}$, and $72^{\circ} \mathrm{C}$ for $45 \mathrm{~s}$, and a final extension at $72^{\circ} \mathrm{C}$ for $10 \mathrm{~min}$; and for TUB, initial denaturation at $95^{\circ} \mathrm{C}$ for $3 \mathrm{~min}$, followed by 35 cycles of $94^{\circ} \mathrm{C}$ for $15 \mathrm{~s}, 58^{\circ} \mathrm{C}$ for $15 \mathrm{~s}$, and $72^{\circ} \mathrm{C}$ for $1 \mathrm{~min}$, and a final extension at $72^{\circ} \mathrm{C}$ for $7 \mathrm{~min}$. All PCRs were carried out in a MyCycler Thermal Cycler (Bio-Rad, Hercules, CA). Amplification products were checked by electrophoresis with a $1.5 \%(\mathrm{w} / \mathrm{v})$ agarose gel stained with RedSafe (Intron Biotechnology, Seongnam-Si, Korea) and visualized under ultraviolet light. A 100-bp DNA Ladder-GTP (gTPbio, C. Viral, Sevilla, Spain) was used as a molecular weight marker. Subsequently, the PCR products were purified using the MEGAquick-spin Total Fragment DNA Purification kit (Intron Biotechnology) following the manufacturer's instructions. The resulting amplicons were sequenced in both directions by the Central Service Support Research of the University of Cordoba (Spain).

Phylogenetic analysis. Consensus sequences were obtained from DNA sequences generated with forward and reverse primers using SeqMan software (DNASTART Lasergen SeqMan version 7.0.0, Madison, WI) and were compiled into a single FASTA file format. To determine whether the sequence datasets (ITS/EF/TUB) were congruent and combinable, a partition-homogeneity test was carried out by PAUP version 4.0b10 (Swofford 2003), and neighbor-joining analysis was performed individually for each locus (data not shown). The neighbor-joining analysis was conducted using the maximum composite likelihood method and 2,000 bootstrap replications, and genetic distances were calculated using the Kimura two-parameter mode. The partition-homogeneity test $(P=0.39)$ was not significant for the combined ITS/EF/TUB data set; consequently, they could be combined. Moreover, tree topologies of $70 \%$ reciprocal bootstrap generated individually for each locus were compared visually, and 
Table 2. Fungal isolates used in the phylogenetic analysis and their corresponding GenBank accession numbers

\begin{tabular}{|c|c|c|c|c|c|c|c|c|}
\hline \multirow[b]{2}{*}{ Species } & \multirow[b]{2}{*}{ Isolate $^{x}$} & \multirow[b]{2}{*}{ Host/cultivar } & \multirow[b]{2}{*}{ Origin } & \multirow[b]{2}{*}{ Collector } & \multirow[b]{2}{*}{ Date $^{\mathbf{y}}$} & \multicolumn{3}{|c|}{ GenBank accession number ${ }^{\mathrm{z}}$} \\
\hline & & & & & & ITS & $\mathbf{E F}$ & TUB \\
\hline \multicolumn{9}{|l|}{$\begin{array}{l}\text { Botryosphaeriaceae } \\
\text { analyses }\end{array}$} \\
\hline \multirow[t]{6}{*}{$\begin{array}{l}\text { Botryosphaeria } \\
\text { dothidea }\end{array}$} & ColPat-443* & $\begin{array}{l}\text { Juglans regia } \mathrm{cv} . \\
\text { Tulare }\end{array}$ & $\begin{array}{l}\text { Benavides, } \\
\text { Badajoz, Spain }\end{array}$ & $\begin{array}{l}\text { C. Agustí-Brisach } \\
\text { \& A. Trapero }\end{array}$ & $07 / 14 / 2017$ & MK431835 & MK461889 & MK522082 \\
\hline & ColPat-446 & J. regia cv. Serr & $\begin{array}{l}\text { Benavides, } \\
\text { Badajoz, Spain }\end{array}$ & $\begin{array}{l}\text { C. Agustí-Brisach } \\
\text { \& A. Trapero }\end{array}$ & $07 / 14 / 2017$ & MK431836 & MK461890 & MK522083 \\
\hline & ColPat-530 & $\begin{array}{l}\text { J. regia cv. } \\
\text { Chandler }\end{array}$ & $\begin{array}{l}\text { Alcalá del Río, } \\
\text { Sevilla, Spain }\end{array}$ & $\begin{array}{l}\text { C. Agustí-Brisach } \\
\text { \& A. Trapero }\end{array}$ & $10 / 18 / 2017$ & MK431843 & MK461897 & MK522084 \\
\hline & ColPat-553 & J. regia cv. Hartley & Badajoz, Spain & $\begin{array}{l}\text { C. Agustí-Brisach } \\
\text { \& A. Trapero }\end{array}$ & $11 / 03 / 2017$ & MK431844 & MK461898 & MK522085 \\
\hline & $\mathrm{CMW8000}^{\mathrm{T}}$ & Prunus sp. & $\begin{array}{l}\text { Crocifisso, } \\
\text { Switzerland }\end{array}$ & B. Slippers & $\mathrm{n} / \mathrm{d}$ & AY236949 & AY236898 & AY236927 \\
\hline & $6 \mathrm{I19}$ & J. regia & Yuba, CA & T. J. Michailides & 09/16/2011 & KF778786 & KF778976 & KF778881 \\
\hline Diplodia juglandis & CBS 188.87 & J. regia & France & $\mathrm{n} / \mathrm{d}$ & $\mathrm{n} / \mathrm{d}$ & EU673316 & EU673283 & EU673119 \\
\hline \multirow[t]{2}{*}{ Diplodia mutila } & CBS $1^{12553}{ }^{T}$ & Vitis vinifera & $\begin{array}{l}\text { Montemor-o- } \\
\text { Novo, Portugal }\end{array}$ & A. J. L. Phillips & $\mathrm{n} / \mathrm{d}$ & AY259093 & AY573219 & DQ458850 \\
\hline & $6 B 99$ & J. regia & Yolo, CA & T. J. Michailides & $05 / 31 / 2011$ & KF778791 & KF778981 & KF778886 \\
\hline \multirow[t]{5}{*}{ Diplodia seriata } & ColPat-366 & J. regia cv. Vina & $\begin{array}{l}\text { Antequera, } \\
\text { Málaga, Spain }\end{array}$ & $\begin{array}{l}\text { C. Agustí-Brisach } \\
\text { \& A. Trapero }\end{array}$ & $04 / 11 / 2017$ & MK431829 & MK461883 & MK522086 \\
\hline & ColPat-367 & J. regia cv. Vina & $\begin{array}{l}\text { Antequera, } \\
\text { Málaga, Spain }\end{array}$ & $\begin{array}{l}\text { C. Agustí-Brisach } \\
\text { \& A. Trapero }\end{array}$ & $04 / 11 / 2017$ & MK431830 & MK461884 & MK522087 \\
\hline & ColPat-557* & J. regia cv. Serr & Badajoz, Spain & $\begin{array}{l}\text { C. Agustí-Brisach } \\
\text { \& A. Trapero }\end{array}$ & $11 / 03 / 2017$ & MK431847 & MK461901 & MK522088 \\
\hline & CBS $1^{112555}{ }^{T}$ & Vitis vinifera & $\begin{array}{l}\text { Montemor-o- } \\
\text { Novo, Portugal }\end{array}$ & A. J. L. Phillips & $\mathrm{n} / \mathrm{d}$ & AY259094 & AY573220 & DQ458856 \\
\hline & $3 \mathrm{H} 18$ & J. regia & $\begin{array}{l}\text { Parlier, Fresno, } \\
\text { CA }\end{array}$ & T. J. Michailides & $01 / 15 / 2004$ & KF778796 & KF778986 & KF778891 \\
\hline \multirow[t]{2}{*}{ Dothiorella iberica } & CBS $115041^{T}$ & Quercus ilex & Spain & J. Luque & $\mathrm{n} / \mathrm{d}$ & AY573202 & AY573222 & EU673096 \\
\hline & 5G97 & J. regia & Davis, Yolo, CA & T. J. Michailides & $12 / 13 / 2010$ & KF778808 & KF778998 & KF778903 \\
\hline $\begin{array}{r}\text { Dothiorella } \\
\text { omnivora }\end{array}$ & CBS 392.80 ${ }^{\mathrm{T}}$ & $\mathrm{n} / \mathrm{d}$ & France & $\mathrm{n} / \mathrm{d}$ & $\mathrm{n} / \mathrm{d}$ & KX464133 & KX464626 & KX464897 \\
\hline \multirow[t]{4}{*}{$\begin{array}{l}\text { Dothiorella } \\
\text { sarmentorum }\end{array}$} & ColPat-469 & $\begin{array}{l}\text { J. regia cv. } \\
\text { Chandler }\end{array}$ & $\begin{array}{l}\text { Chirivel, Almería, } \\
\text { Spain }\end{array}$ & $\begin{array}{l}\text { C. Agustí-Brisach } \\
\text { \& A. Trapero }\end{array}$ & $07 / 27 / 2017$ & MK431838 & MK461892 & MK522089 \\
\hline & ColPat-471* & $\begin{array}{l}\text { J. regia cv. } \\
\text { Chandler }\end{array}$ & $\begin{array}{l}\text { Chirivel, Almería, } \\
\text { Spain }\end{array}$ & $\begin{array}{l}\text { C. Agustí-Brisach } \\
\text { \& A. Trapero }\end{array}$ & $07 / 27 / 2017$ & MK431840 & MK461894 & MK522090 \\
\hline & IMI $63581 b^{T}$ & Ulmus sp. & $\begin{array}{l}\text { Warwickshire, } \\
\text { U.K. }\end{array}$ & E. A. Ellis & $\mathrm{n} / \mathrm{d}$ & AY573212 & AY573235 & EU673102 \\
\hline & CBS 115038 & Malus pumila & $\begin{array}{l}\text { Delft, The } \\
\text { Netherlands }\end{array}$ & A. J. L. Phillips & $\mathrm{n} / \mathrm{d}$ & AY573206 & AY573223 & EU673101 \\
\hline \multirow[t]{3}{*}{ Dothiorella sp. } & ColPat-370 & J. regia cv. Vina & $\begin{array}{l}\text { Antequera, } \\
\text { Málaga, Spain }\end{array}$ & $\begin{array}{l}\text { C. Agustí-Brisach } \\
\text { \& A. Trapero }\end{array}$ & $04 / 11 / 2017$ & MK431833 & MK461887 & MK522091 \\
\hline & ColPat-371 & J. regia cv. Vina & $\begin{array}{l}\text { Antequera, } \\
\text { Málaga, Spain }\end{array}$ & $\begin{array}{l}\text { C. Agustí-Brisach } \\
\text { \& A. Trapero }\end{array}$ & $04 / 11 / 2017$ & MK431834 & MK461888 & MK522092 \\
\hline & ColPat- $470 *$ & $\begin{array}{l}\text { J. regia cv. } \\
\text { Chandler }\end{array}$ & $\begin{array}{l}\text { Antequera, } \\
\text { Málaga, Spain }\end{array}$ & $\begin{array}{l}\text { C. Agustí-Brisach } \\
\text { \& A. Trapero }\end{array}$ & $04 / 11 / 2017$ & MK431839 & MK461893 & MK522093 \\
\hline $\begin{array}{l}\text { Dothiorella } \\
\text { vidmadera }\end{array}$ & CBS 725.79 & $\mathrm{n} / \mathrm{d}$ & $\mathrm{n} / \mathrm{d}$ & $\mathrm{n} / \mathrm{d}$ & $\mathrm{n} / \mathrm{d}$ & KX464130 & KX464622 & KX464888 \\
\hline $\begin{array}{l}\text { Dothiorella } \\
\quad \text { viticola }\end{array}$ & CBS 117009 & $\begin{array}{l}\text { Vitis vinifera cv. } \\
\text { Garnatxa Negra }\end{array}$ & Spain & $\mathrm{n} / \mathrm{d}$ & $\mathrm{n} / \mathrm{d}$ & KF766228 & AY905559 & EU673104 \\
\hline \multirow[t]{2}{*}{$\begin{array}{l}\text { Lasiodiplodia } \\
\text { citricola }\end{array}$} & $\begin{array}{l}\text { CBS } 124707^{\mathrm{T}} \\
\text { IRAN1522C }\end{array}$ & Citrus sp. & Iran & $\begin{array}{l}\text { J. Adbollahzadeh } \\
\text { \& A. Javadi }\end{array}$ & $\mathrm{n} / \mathrm{d}$ & GU945354 & GU945340 & KU887505 \\
\hline & 6134 & J. regia & Stanislaus, CA & T. J. Michailides & $10 / 06 / 2011$ & KF778809 & KF778999 & KF778904 \\
\hline \multirow[t]{2}{*}{$\begin{array}{r}\text { Lasiodiplodia } \\
\text { theobromae }\end{array}$} & CBS $164.96^{T}$ & $\begin{array}{l}\text { Fruit on coral reef } \\
\text { coast }\end{array}$ & New Guinea & A. Aptroot & $\mathrm{n} / \mathrm{d}$ & AY640255 & AY640258 & EU673110 \\
\hline & CBS 124.13 & $\mathrm{n} / \mathrm{d}$ & U.S.A. & J. J. Taubenhau & $\mathrm{n} / \mathrm{d}$ & DQ458890 & DQ458875 & DQ458858 \\
\hline \multirow[t]{3}{*}{$\begin{array}{l}\text { Neofusicoccum } \\
\text { mediterraneum }\end{array}$} & ColPat-368 & J. regia cv. Vina & $\begin{array}{l}\text { Antequera, } \\
\text { Málaga, Spain }\end{array}$ & $\begin{array}{l}\text { C. Agustí-Brisach } \\
\text { \& A. Trapero }\end{array}$ & $04 / 11 / 2017$ & MK431831 & MK461885 & MK522094 \\
\hline & ColPat-369 & J. regia cv. Vina & $\begin{array}{l}\text { Antequera, } \\
\text { Málaga, Spain }\end{array}$ & $\begin{array}{l}\text { C. Agustí-Brisach } \\
\text { \& A. Trapero }\end{array}$ & $04 / 11 / 2017$ & MK431832 & MK461886 & MK522095 \\
\hline & & & & & & & \multicolumn{2}{|c|}{ (Continued on next page) } \\
\hline
\end{tabular}

${ }^{x}$ Sequences from GenBank used in the phylogenetic analysis indicated in bold type. $\mathrm{T}=$ ex-type isolates; $\mathrm{AR}$ and $\mathrm{DP}=$ isolates in culture collection of the Systematic Mycology and Microbiology Laboratory, USDA-ARS, Beltsville, MD, U.S.A.; CAA = A. Alves, Universidade de Aveiro, Portugal; CBS = culture collection of the Centraalbureau voor Schimmelcultures, Fungal Biodiversity Centre, Utrecht, The Netherlands; CMW = culture collection of the Forestry and Agricultural Biotechnology Institute (FABI), University of Pretoria, Pretoria, South Africa; ColPat = "Colección Patología", Department of Agronomy, University of Cordoba, Spain; IMI = CABI Bioscience, Egham, U.K.; IRAN = Department of Botany, Iranian Research Institute of Plant Protection, Tehran, Iran; and PD = Plant Protection Service, Wageningen, The Netherlands. Asterisk $(*)$ indicates isolates selected for morphological characterization, study of the effect of temperature on mycelial growth, and pathogenicity tests.

${ }^{\mathrm{y}}$ Collection date $=$ month/day/year; and $\mathrm{n} / \mathrm{d}=$ nondetermined.

${ }^{\mathrm{z}}$ ITS = internal transcribed spacer; $\mathrm{EF}=$ translation elongation $1-\alpha ;$ and TUB $=\beta$-tubulin- 2 gene regions. 
Table 2. (Continued from previous page)

\begin{tabular}{|c|c|c|c|c|c|c|c|c|}
\hline \multirow[b]{2}{*}{ Species } & \multirow[b]{2}{*}{ Isolate $^{x}$} & \multirow[b]{2}{*}{ Host/cultivar } & \multirow[b]{2}{*}{ Origin } & \multirow[b]{2}{*}{ Collector } & \multirow[b]{2}{*}{ Date $^{y}$} & \multicolumn{3}{|c|}{ GenBank accession number ${ }^{z}$} \\
\hline & & & & & & ITS & EF & TUB \\
\hline & ColPat-556* & J. regia cv. Vina & Badajoz, Spain & $\begin{array}{l}\text { C. Agustí-Brisach } \\
\text { \& A. Trapero }\end{array}$ & $11 / 03 / 2017$ & MK431846 & MK461900 & MK522096 \\
\hline & ColPat-561 & $\begin{array}{l}\text { J. regia cv. } \\
\text { Chandler }\end{array}$ & Badajoz, Spain & $\begin{array}{l}\text { C. Agustí-Brisach } \\
\text { \& A. Trapero }\end{array}$ & $11 / 03 / 2017$ & MK431849 & MK461903 & MK522097 \\
\hline & $\begin{array}{l}\text { CBS 121718 }^{\mathrm{T}} \text {; } \\
\text { PD312 }\end{array}$ & Eucalyptus sp. & Greece & $\begin{array}{l}\text { P. W. Crous, M. J. } \\
\text { Wingfield, \& } \\
\text { A. J. L. Phillips }\end{array}$ & $\mathrm{n} / \mathrm{d}$ & GU251176 & GU251308 & GU251836 \\
\hline & $1 \mathrm{H} 96$ & J. regia & Selma, Fresno, CA & T. J. Michailides & $09 / 15 / 2006$ & KF778811 & KF779001 & KF778906 \\
\hline & CAA002 & $\begin{array}{l}\text { Pistacia vera } \mathrm{cv} . \\
\text { Kerman }\end{array}$ & U.S.A. & T. J. Michailides & $\mathrm{n} / \mathrm{d}$ & EU017537 & EU017538 & KX505925 \\
\hline & Bot-04 & $\begin{array}{l}\text { Vitis vinifera cv. } \\
\text { Pedro Ximénez }\end{array}$ & $\begin{array}{c}\text { Montilla, Córdoba, } \\
\text { Andalusia, Spain }\end{array}$ & $\begin{array}{l}\text { C. Agustí-Brisach } \\
\text { \& A. Trapero }\end{array}$ & 2016 & MG745841 & MG745817 & MG745803 \\
\hline \multirow[t]{9}{*}{$\begin{array}{l}\text { Neofusicoccum } \\
\text { parvum }\end{array}$} & ColPat-447 & J. regia cv. Serr & $\begin{array}{l}\text { Benavides, } \\
\text { Badajoz, Spain }\end{array}$ & $\begin{array}{l}\text { C. Agustí-Brisach } \\
\text { \& A. Trapero }\end{array}$ & $07 / 14 / 2017$ & MK431837 & MK461891 & MK522098 \\
\hline & ColPat-526 & $\begin{array}{l}\text { J. regia cv. } \\
\text { Chandler }\end{array}$ & $\begin{array}{l}\text { Alcalá del Río, } \\
\text { Sevilla, Spain }\end{array}$ & $\begin{array}{l}\text { C. Agustí-Brisach } \\
\text { \& A. Trapero }\end{array}$ & $10 / 18 / 2017$ & MK431841 & MK461895 & MK522099 \\
\hline & ColPat-528 & $\begin{array}{l}\text { J. regia cv. } \\
\text { Chandler }\end{array}$ & $\begin{array}{l}\text { Alcalá del Río, } \\
\text { Sevilla, Spain }\end{array}$ & $\begin{array}{l}\text { C. Agustí-Brisach } \\
\text { \& A. Trapero }\end{array}$ & $10 / 18 / 2017$ & MK431842 & MK461896 & MK522100 \\
\hline & ColPat-554* & J. regia cv. Hartley & Badajoz, Spain & $\begin{array}{l}\text { C. Agustí-Brisach } \\
\text { \& A. Trapero }\end{array}$ & $11 / 03 / 2017$ & MK431845 & MK461899 & MK522101 \\
\hline & ColPat-558 & J. regia cv. Serr & Badajoz, Spain & $\begin{array}{l}\text { C. Agustí-Brisach } \\
\text { \& A. Trapero }\end{array}$ & $11 / 03 / 2017$ & MK431848 & MK461902 & MK522102 \\
\hline & ColPat-562 & $\begin{array}{l}\text { J. regia cv. } \\
\text { Chandler }\end{array}$ & Badajoz, Spain & $\begin{array}{l}\text { C. Agustí-Brisach } \\
\text { \& A. Trapero }\end{array}$ & $11 / 03 / 2017$ & MK431850 & MK461904 & MK522103 \\
\hline & ColPat-563 & $\begin{array}{l}\text { J. regia cv. } \\
\text { Chandler }\end{array}$ & Badajoz, Spain & $\begin{array}{l}\text { C. Agustí-Brisach } \\
\text { \& A. Trapero }\end{array}$ & $11 / 03 / 2017$ & MK431851 & MK461905 & MK522104 \\
\hline & CMW9081 $^{\mathrm{T}}$ & Pinus nigra & New Zealand & G. J. Samuels & $\mathrm{n} / \mathrm{d}$ & AY236943 & AY236888 & AY236917 \\
\hline & $1 \mathrm{L83}$ & J. regia & Butte, CA & T. J. Michailides & $11 / 01 / 2005$ & KF778854 & KF779044 & KF778949 \\
\hline $\begin{array}{l}\text { Neoscytalidium } \\
\text { dimidiatum }\end{array}$ & CBS 499.66 & Mangifera indica & Mali & $\mathrm{n} / \mathrm{d}$ & $\mathrm{n} / \mathrm{d}$ & FM211432 & EU144063 & FM211167 \\
\hline \multicolumn{9}{|l|}{ Diaporthe analyses } \\
\hline $\begin{array}{l}\text { Diaporthe } \\
\text { acaciigena }\end{array}$ & CBS ${ }^{129521}{ }^{T}$ & Acacia retinodes & Australia & $\begin{array}{l}\text { P. W. Crous, I. G. } \\
\text { Pascoe, \& J. } \\
\text { Edwards }\end{array}$ & $\mathrm{n} / \mathrm{d}$ & KC343005 & KC343731 & KC343973 \\
\hline $\begin{array}{l}\text { Diaporthe } \\
\text { alleghaniensis }\end{array}$ & CBS $495.72^{T}$ & $\begin{array}{l}\text { Betula } \\
\text { alleghaniensis }\end{array}$ & Ontario, Canada & R. H. Arnold & $\mathrm{n} / \mathrm{d}$ & FJ889444 & GQ250298 & KC343975 \\
\hline Diaporthe alnea & CBS 146.46 & Alnus sp. & $\mathrm{n} / \mathrm{d}$ & S. Truter & $\mathrm{n} / \mathrm{d}$ & KC343008 & KC343734 & KC343976 \\
\hline Diaporthe arctii & DP0482 & Arctium lappa & Austria & W. Jaklitsch & $\mathrm{n} / \mathrm{d}$ & KJ590736 & KJ590776 & KJ610891 \\
\hline $\begin{array}{l}\text { Diaporthe } \\
\text { asheicola }\end{array}$ & CBS 136967 & Vaccinium ashei & Chile & A. Schilder & $\mathrm{n} / \mathrm{d}$ & KJ160562 & KJ160594 & KJ160518 \\
\hline $\begin{array}{l}\text { Diaporthe } \\
\quad \text { australafricana }\end{array}$ & CBS 111886 $^{T}$ & Vitis vinifera & Australia & R. W. A. Schepers & $\mathrm{n} / \mathrm{d}$ & KC343038 & КC343764 & KC344006 \\
\hline \multirow[t]{2}{*}{$\begin{array}{l}\text { Diaporthe } \\
\text { chamaeropis }\end{array}$} & CBS 454.81 & $\begin{array}{l}\text { Chamaerops } \\
\text { humilis, }\end{array}$ & Greece & H. A. van der Aa & $\mathrm{n} / \mathrm{d}$ & KC343048 & KC343774 & KC344016 \\
\hline & CBS 753.70 & Spartium junceum & Croatia & J. A. von Arx & $\mathrm{n} / \mathrm{d}$ & KC343049 & КC343775 & KC344017 \\
\hline $\begin{array}{l}\text { Diaporthe } \\
\text { cinerascens }\end{array}$ & CBS 719.96 & Ficus carica & Bulgaria & E. Ilieva & $\mathrm{n} / \mathrm{d}$ & KC343050 & KC343776 & KC344018 \\
\hline $\begin{array}{l}\text { Diaporthe } \\
\text { hickoriae }\end{array}$ & CBS $145.26^{T}$ & Carya glabra & Michigan, U.S.A. & L. E. Wehmeyer & $\mathrm{n} / \mathrm{d}$ & FJ889446 & GQ250309 & KC344086 \\
\hline $\begin{array}{l}\text { Diaporthe } \\
\text { inconspicua }\end{array}$ & CBS 133813 $^{\mathrm{T}}$ & Maytenus ilicifolia & Brazil & R. R. Gomes & $\mathrm{n} / \mathrm{d}$ & KC343123 & КC343849 & KC344091 \\
\hline \multirow[t]{8}{*}{$\begin{array}{l}\text { Diaporthe } \\
\text { neotheicola }\end{array}$} & ColPat-445 & $J$. regia $\mathrm{cv}$. Tulare & $\begin{array}{l}\text { Benavides, } \\
\text { Badajoz, Spain }\end{array}$ & $\begin{array}{l}\text { C. Agustí-Brisach } \\
\text { \& A. Trapero }\end{array}$ & $07 / 14 / 2017$ & MK447993 & MK490932 & MK522106 \\
\hline & ColPat- $448 *$ & J. regia cv. Serr & $\begin{array}{l}\text { Benavides, } \\
\text { Badajoz, Spain }\end{array}$ & $\begin{array}{l}\text { C. Agustí-Brisach } \\
\text { \& A. Trapero }\end{array}$ & $07 / 14 / 2017$ & MK447994 & MK490939 & MK522107 \\
\hline & ColPat-450 & J. regia cv. Vina & Córdoba, Spain & $\begin{array}{l}\text { C. Agustí-Brisach } \\
\text { \& A. Trapero }\end{array}$ & $07 / 14 / 2017$ & MK447996 & MK490934 & MK522109 \\
\hline & ColPat-451 & J. regia cv. Vina & Córdoba, Spain & $\begin{array}{l}\text { C. Agustí-Brisach } \\
\text { \& A. Trapero }\end{array}$ & $07 / 14 / 2017$ & MK447997 & MK490935 & MK522110 \\
\hline & ColPat-532 & $\begin{array}{l}\text { J. regia cv. } \\
\text { Chandler }\end{array}$ & $\begin{array}{l}\text { Alcalá del Río, } \\
\text { Sevilla, Spain }\end{array}$ & $\begin{array}{l}\text { C. Agustí-Brisach } \\
\text { \& A. Trapero }\end{array}$ & $10 / 18 / 2017$ & MK447998 & MK490936 & MK522111 \\
\hline & ColPat-551 & J. regia cv. Hartley & Badajoz, Spain & $\begin{array}{l}\text { C. Agustí-Brisach } \\
\text { \& A. Trapero }\end{array}$ & $11 / 03 / 2017$ & MK448000 & MK490940 & MK522112 \\
\hline & ColPat-552 & J. regia cv. Hartley & Badajoz, Spain & $\begin{array}{l}\text { C. Agustí-Brisach } \\
\text { \& A. Trapero }\end{array}$ & $11 / 03 / 2017$ & MK448001 & MK490938 & MK522113 \\
\hline & ColPat-555 & J. regia cv. Serr & Badajoz, Spain & $\begin{array}{l}\text { C. Agustí-Brisach } \\
\text { \& A. Trapero }\end{array}$ & $11 / 03 / 2017$ & MK448002 & MK490941 & MK522114 \\
\hline
\end{tabular}


the data of different loci were combined into single concatenated datasets because no supported nodes were in conflict.

Two separate phylogenetic analyses were conducted to identify Botryosphaeriaceae and Diaporthe species. To infer the phylogeny of isolates belonging to Botryosphaeriaceae, the combined alignment of the ITS, EF, and TUB loci was analyzed. In this analysis, a total of 47 taxa were included, of which 23 were the representative isolates of Botryosphaeriaceae used in this study, 23 were GenBank taxa selected based on their high similarity with our query sequences using MegaBLAST, and one was Neo. dimidiatum isolate CBS 499.66, which was used as an outgroup (Table 2). In the case of Diaporthe isolates, the combined alignment of the TUB and EF loci was conducted $(P=0.56)$. In this analysis, a total of 34 taxa sequences were included, of which 13 were the representative isolates of Diaporthe used in this study, 20 were GenBank sequences selected based on their high similarity with our query sequences using MegaBLAST, and one was Diaporthella corylina isolate CBS 121124, which was used as an outgroup (Table 2).

In both cases, GenBank sequences were added to the sequences obtained and aligned by Clustal W. Subsequently, maximum parsimony (MP) analyses by the tree-bisection-regrafting algorithm with search level one in which the initial trees were obtained by the random addition of sequences (10 replicates). Adding sequences, alignments, and MP analyses were performed using MEGA version 7.0 software (Kumar et al. 2016). Gaps and missing data were treated as complete deletions. The robustness of the topology was evaluated by 2,000 bootstrap replications (Felsenstein 1985). Measurements for the MP analyses, namely, tree length (TL), consistency index $(\mathrm{CI})$, retention index $(\mathrm{RI})$, homoplasy index $(\mathrm{HI})$, and rescaled consistency index (RC) were calculated for the resulting tree. Additionally, Bayesian inference (BI) analyses were performed by MrBayes version 3.2.6 (Ronquist et al. 2012) using Markov chain Monte Carlo (MCMC) methods to estimate the posterior probability of trees. Models of nucleotide substitution used for each gene partition were also determined by MEGA version 7.0 (Kumar et al. 2016) and included in the analyses. Two analyses with four MCMC chains each were run simultaneously for $1 \times 10^{7}$ generations, starting from a random tree topology. Trees were sampled every 100 generations, and the "temperature" parameter was set to 0.2 . The first $25 \%$ of the saved trees was discarded as the burn-in phase of the analysis. Finally, the ITS, EF, and TUB sequences of Cytospora spp. isolates were directly BLAST searched in GenBank (https://www.ncbi.nlm.nih.gov/genbank/). Sequences derived in this study were lodged at GenBank, and their GenBank accession numbers are shown in Table 2.

Pathogenicity tests. Fungal isolates. All inoculations were performed using isolates of Botryosphaeriaceae, Diaporthe, and Cytospora species (10 isolates in total were selected; Table 2). For inoculum preparation, all the isolates were grown on PDA at $25^{\circ} \mathrm{C}$ in darkness for 10 days.

Inoculation of detached shoots. In April 2018, 1-year-old detached shoots of walnut cv. Chandler were collected from a 10 -year-old experimental orchard belonging to the Andalusian Institute for Research and Formation in Agriculture and Fishery (IFAPA in Spanish), located in Alcalá del Río (Sevilla Province, Andalusia region). Branch segments approximately $25 \mathrm{~cm}$ in length were cut and immediately sealed at both ends with Parafilm (Parafilm, Menasha, WI) to reduce desiccation. Subsequently, they were maintained at $4^{\circ} \mathrm{C}$ until they were processed in the laboratory. Prior to inoculation, the bark surface was cleaned in a water bath with $0.02 \%$ Tween 20 for $1 \mathrm{~min}$, surface disinfected for $1 \mathrm{~min}$ in $20 \%$ sodium hypochlorite solution, washed twice with distilled water, and air dried on sterile filter paper for $30 \mathrm{~min}$. The outer bark was removed from the center of the branch segment using a 7.5-mm-diameter cork borer, and branch segments were placed in humid chambers (plastic containers, $32 \times 25 \times$ $8 \mathrm{~cm}$ ). Subsequently, they were inoculated with a 7.5-mm-diameter mycelium plug obtained from the actively growing margin of the fungal colony, which was deposited in contact with the wounded tissue, and the inoculated point was immediately wrapped with Parafilm. Inoculated shoots were incubated at $27^{\circ} \mathrm{C}$ and $100 \%$ relative humidity for 3 weeks. There were three replicated humid chambers per fungal

Table 2. (Continued from previous page)

\begin{tabular}{|c|c|c|c|c|c|c|c|c|}
\hline \multirow[b]{2}{*}{ Species } & \multirow[b]{2}{*}{ Isolate $^{x}$} & \multirow[b]{2}{*}{ Host/cultivar } & \multirow[b]{2}{*}{ Origin } & \multirow[b]{2}{*}{ Collector } & \multirow[b]{2}{*}{ Date $^{\mathbf{y}}$} & \multicolumn{3}{|c|}{ GenBank accession number ${ }^{\mathrm{z}}$} \\
\hline & & & & & & ITS & $\mathbf{E F}$ & TUB \\
\hline & ColPat-559 & $\begin{array}{l}\text { J. regia } \mathrm{cv} . \\
\text { Chandler }\end{array}$ & Badajoz, Spain & $\begin{array}{l}\text { C. Agustí-Brisach } \\
\text { \& A. Trapero }\end{array}$ & $11 / 03 / 2017$ & MK448003 & MK490942 & MK522115 \\
\hline & ColPat-560 & $\begin{array}{l}\text { J. regia cv. } \\
\text { Chandler }\end{array}$ & Badajoz, Spain & $\begin{array}{l}\text { C. Agustí-Brisach } \\
\text { \& A. Trapero }\end{array}$ & $11 / 03 / 2017$ & MK448004 & MK490943 & MK522116 \\
\hline & CBS 123208 & $\begin{array}{l}\text { Foeniculum } \\
\text { vulgare }\end{array}$ & Vora, Portugal & A. J. L. Phillips & $\mathrm{n} / \mathrm{d}$ & EU814480 & GQ250315 & KC344072 \\
\hline & CBS $111553^{T}$ & F. vulgare & Spain & A. J. L. Phillips & $\mathrm{n} / \mathrm{d}$ & KC343101 & KC343827 & KC344069 \\
\hline & $6 \mathbf{I 3 0}$ & J. regia & Stanislaus, CA & T. J. Michailides & $10 / 06 / 2011$ & KF778871 & KF779061 & KF778966 \\
\hline $\begin{array}{l}\text { Diaporthe } \\
\text { phaseolorum }\end{array}$ & AR4203 & Phaseolus vulgaris & U.S.A. & N. F. Gregory & $\mathrm{n} / \mathrm{d}$ & KJ590738 & KJ590739 & KJ610893 \\
\hline \multirow[t]{4}{*}{$\begin{array}{l}\text { Diaporthe } \\
\text { rhusicola }\end{array}$} & ColPat-444* & J. regia cv. Tulare & $\begin{array}{l}\text { Benavides, } \\
\text { Badajoz, Spain }\end{array}$ & $\begin{array}{l}\text { C. Agustí-Brisach } \\
\text { \& A. Trapero }\end{array}$ & $07 / 14 / 2017$ & MK447992 & MK490931 & MK522105 \\
\hline & $6 \mathrm{I15}$ & Prunus dulcis & Kings, CA & T. J. Michailides & $09 / 12 / 2011$ & KF778873 & KF779063 & KF778968 \\
\hline & $6 \mathrm{I31}$ & J. regia & Stanislaus, CA & T. J. Michailides & $10 / 06 / 2011$ & KF778874 & KF779064 & KF778969 \\
\hline & $6 \mathbf{I 4 3}$ & J. regia & Stanislaus, CA & T. J. Michailides & $10 / 06 / 2011$ & KF778875 & KF779065 & KF778970 \\
\hline Diaporthe sp. & ColPat-449 & J. regia cv. Serr & $\begin{array}{l}\text { Benavides, } \\
\text { Badajoz, Spain }\end{array}$ & $\begin{array}{l}\text { C. Agustí-Brisach } \\
\text { \& A. Trapero }\end{array}$ & $07 / 27 / 2017$ & MK447995 & MK490933 & MK522108 \\
\hline $\begin{array}{l}\text { Diaporthella } \\
\text { corylina }\end{array}$ & CBS 121124 & Corylus sp. & Fuyuan, China & L. N. Vassiljeva & $\mathrm{n} / \mathrm{d}$ & KC343004 & KC343730 & KC343972 \\
\hline \multirow[t]{3}{*}{$\begin{array}{l}\text { Phomopsis } \\
\text { amygdali }\end{array}$} & ColPat-533* & $\begin{array}{l}\text { J. regia } \mathrm{cv} . \\
\text { Chandler }\end{array}$ & $\begin{array}{l}\text { Alcalá del Río, } \\
\text { Sevilla, Spain }\end{array}$ & $\begin{array}{l}\text { C. Agustí-Brisach } \\
\text { \& A. Trapero }\end{array}$ & $10 / 18 / 2017$ & MK447999 & MK490937 & MK522117 \\
\hline & CBS 126679T & P. dulcis & Portugal & E. Diogo & $\mathrm{n} / \mathrm{d}$ & KC343022 & KC343748 & KC343990 \\
\hline & CBS 115620 & Prunus persica & Georgia, U.S.A. & W. Uddin & $\mathrm{n} / \mathrm{d}$ & KC343020 & KC343746 & KC343988 \\
\hline \multicolumn{9}{|l|}{ Cytospora BLAST } \\
\hline \multirow[t]{3}{*}{ Cytospora sp. } & ColPat-465* & $\begin{array}{l}\text { J. regia } \mathrm{cv} . \\
\text { Chandler }\end{array}$ & $\begin{array}{l}\text { Guadix, Granada, } \\
\text { Spain }\end{array}$ & $\begin{array}{l}\text { C. Agustí-Brisach } \\
\text { \& A. Trapero }\end{array}$ & $07 / 26 / 2017$ & MK480242 & MK490946 & MK546589 \\
\hline & ColPat-633 & $\begin{array}{l}\text { J. regia } \mathrm{cv} . \\
\text { Chandler }\end{array}$ & $\begin{array}{l}\text { Guadix, Granada, } \\
\text { Spain }\end{array}$ & $\begin{array}{l}\text { C. Agustí-Brisach } \\
\text { \& A. Trapero }\end{array}$ & $07 / 19 / 2018$ & MK480241 & MK490944 & MK546587 \\
\hline & ColPat-634 & $\begin{array}{l}\text { J. regia } \mathrm{cv} . \\
\text { Chandler }\end{array}$ & $\begin{array}{l}\text { Guadix, Granada, } \\
\text { Spain }\end{array}$ & $\begin{array}{l}\text { C. Agustí-Brisach } \\
\text { \& A. Trapero }\end{array}$ & $07 / 19 / 2018$ & MK480240 & MK490945 & MK546588 \\
\hline
\end{tabular}


isolate and five shoots per humid chamber. Fifteen additional shoots (three humid chambers, five shoots in each) treated with 7.5mm-diameter PDA plugs were included as controls. A completely randomized block design was used with the fungal isolate as the independent variable, the shoots as replications, and the humid chambers as blocks. For each treatment (inoculated isolates and control), the lesion length of each shoot was recorded at 3 weeks after inoculation, and the measures of the five replicated shoots of each block (humid chamber) was averaged. Per humid chamber, one inoculated or noninoculated control shoot was selected for reisolations, which were performed from the margin of the necrotic lesions of inoculated shoots or from the wounded area of control shoots. Reisolations were conducted onto APDA as described above, and the percentage of reisolation was calculated.
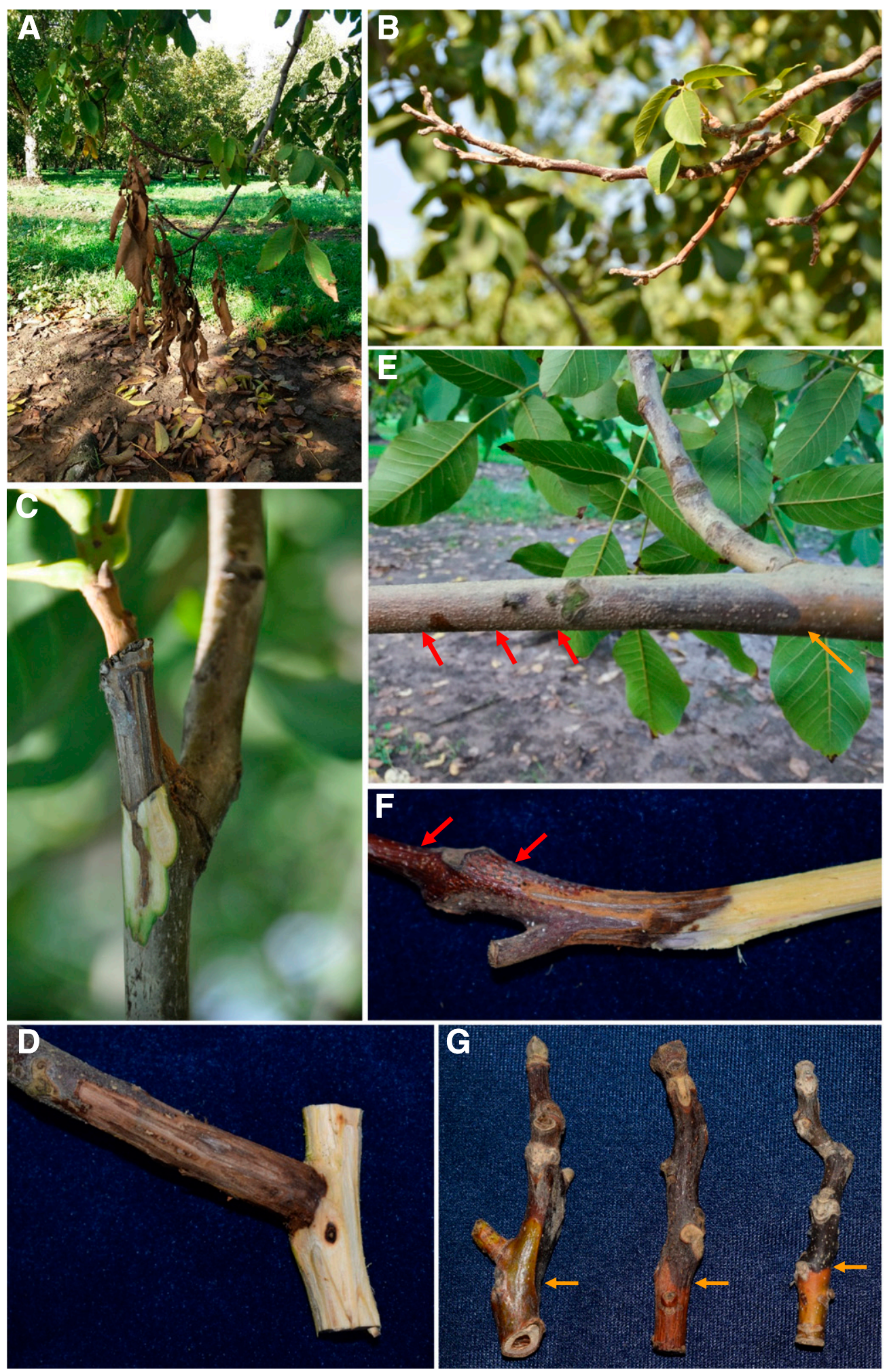

Fig. 1. Symptoms of branch dieback and shoot blight of English walnut in southern Spain. A, Decayed branch showing necrotic foliage; B, typical shoot blight symptoms on young shoots; $\mathbf{C}$ to G, detailed disease progression on shoots and branches showing discoloration on the affected wound (orange arrows); arrows in E and $F$ point to development of pycnidia on the affected areas of the shoots. 
Inoculation of attached shoots under field conditions. One-yearold shoots of walnut cv. Chandler were selected from the same experimental orchard described above. The area of the inoculation was surface disinfected by spraying with $70 \%$ ethanol solution, and inoculations were conducted with mycelial plugs as described above. Inoculation was performed on April 19, 2018. Six walnut trees were randomly selected, and two shoots per isolate were inoculated in each tree (one shoot in the north face and the second one in the south face of the tree canopy), with 12 inoculated shoots per isolate and 120 shoots in total. Additionally, 12 shoots (two branches per replicated tree) were treated with 7.5-mm-diameter PDA plugs as a control. A completely randomized block design was used with fungal isolates as the independent variable, the shoots as replications, and the trees as blocks. For each treatment (inoculated isolates and control), the lesion length of each shoot was recorded at 3 months after inoculation (July 19, 2018), and the measures of the two replicated shoots of each block (tree) were averaged. Three inoculated shoots per isolate and three noninoculated control shoots were selected for reisolations, which were performed as described above. Additionally, reisolations from asymptomatic tissues $2 \mathrm{~cm}$ beyond the margin of the lesion or the wounded area from both inoculated and control shoots were also performed. All the reisolations were conducted onto APDA as described above, and the percentage of reisolation in each tissue was calculated.

Inoculation of detached walnut fruits. Green fruits of walnut cv. Chandler were collected from the same experimental orchard described above. Fruits were surface disinfested following the same protocol described for detached shoots. Disinfested walnut fruits were placed in humid chambers, and a wound of 5-mm diameter $\times$ 4-mm depth was made on the center of the hull of each fruit using a cork borer. Inoculations were conducted by placing a 5-mmdiameter mycelial plug inside the hole. There were three replicated humid chambers per treatment, 10 fruits per humid chamber, 30 fruits per treatment, and 300 fruits in total. Additionally, 30 fruits (three humid chambers, 10 fruits in each) treated with a 5-mm-diameter PDA plug were included as controls. A completely randomized block design was used with fungal isolates as the independent variable, the fruits as replications, and the humid chambers as blocks. Disease severity was evaluated every 3 days until most of the fruits inoculated with the most aggressive isolate had 90 to $100 \%$ of their surface affected. The major and minor diameters of each lesion were recorded and averaged for each evaluation, and the percentage of the affected fruit surface of each lesion in each treatment and evaluation was calculated. The relative area under the disease progress curve (RAUDPC) was obtained by trapezoidal integration (Campbell and Madden 1990) for each inoculated or control fruit, and the RAUDPC values of the 10 fruits of each block (humid chamber) were averaged. Fungal reisolation was conducted from the margin of the necrotic lesions as described above. Additionally, reisolations from the affected or asymptomatic tissues around the peduncle were also performed from both inoculated and control fruits. All the reisolations were conducted onto APDA as described above, and the percentage of reisolation in each tissue was calculated.

Data analysis. The dependent variables lesion length and RAUDPC were subjected to ANOVA to determine the differences in aggressiveness between fungal isolates on infected shoots and green fruits of walnut, respectively. In all cases, data were tested for normality and homogeneity of variances and logarithmically transformed when necessary. In all cases, control treatments were excluded from the statistical analysis because no lesions were observed in noninoculated control shoots or green fruits, and treatment means were compared according to Tukey's HSD test at $\alpha=0.05$ (Steel and Torrie 1985). The Pearson correlation coefficient ( $r$ ) between the lesion length and diameter of the shoots was calculated using the average of the two measures for the fungal isolates evaluated $(n=10)$. Additionally, Pearson's linear correlation was also conducted to compare infections between inoculated detached and attached shoots and detached shoots and fruits using the average lesion length or RAUDPC values for infected shoots and fruits, respectively, for all isolates evaluated $(n=10)$. All data of this study were analyzed using Statistix 10 software (Analytical Software 2013).

\section{Results}

Field surveys and fungal isolation. Typical dieback symptoms, including twig and leaf blight, were observed in association with shoot blight and wood canker formation with internal discoloration (Fig. 1). Fungal fruiting structures were usually found on affected tissues (Fig. 1E and F). From the samples collected during the field surveys, 470 out of 630 attempts of isolation from symptomatic tissues yielded a fungal culture (74.6\%). The isolation of Botryosphaeriaceae fungi was the most consistent with $53.0 \%$ positive inoculation points, followed by Diaporthe fungi with $38.0 \%$ positive inoculation points. These two groups of fungi were recovered alone or simultaneously from seven out of the 10 surveyed orchards. The cooccurrence of Botryosphaeriaceae and Diaporthe fungi was observed in five out of 10 surveyed orchards. Cytospora sp. was isolated alone from a single orchard (Guadix, Granada) with a low frequency of isolation $(9.0 \%)$.

Fungal colony, conidia, and pycnidia morphology. According to their appearance in culture (texture, density, color, zonation, margin, and growth rate), the isolates were first assigned to three main fungal groups. The first group comprised colonies associated with typical characteristics of Botryosphaeriaceae. In general, all isolates of this first group showed floccose to felted fast-growing aerial

Table 3. Morphological characteristics of the representative fungal isolates collected from English walnut showing branch dieback and shoot blight in southern Spain

\begin{tabular}{|c|c|c|c|c|c|c|}
\hline \multirow[b]{3}{*}{ Family/fungal species } & \multirow[b]{3}{*}{ Isolate $^{\mathrm{z}}$} & \multicolumn{5}{|c|}{ Mycelia } \\
\hline & & \multicolumn{3}{|c|}{ Obverse } & \multicolumn{2}{|l|}{ Reverse } \\
\hline & & Color & Zonation & Margin & Color & Zonation \\
\hline \multicolumn{7}{|l|}{ Botryosphaeriaceae } \\
\hline Botryosphaeria dothidea & ColPat-443 & White gray & No & Irregular & Dark to olive green & No \\
\hline Diplodia seriata & ColPat-557 & Light gray-brown & No & Irregular & Light to dark gray & No \\
\hline Dothiorella sarmentorum & ColPat-471 & Dark to olive brown & Yes & Irregular & Dark gray to olive green & Yes \\
\hline Dothiorella sp. & ColPat-470 & Dark gray to olive green & No & Regular & Dark gray to olive green & No \\
\hline Neofusicoccum mediterraneum & ColPat-556 & Light gray to olive green & No & Irregular & Olive green & No \\
\hline Neofusicoccum parvum & ColPat-554 & White to light gray & No & Regular & White to light gray & No \\
\hline \multicolumn{7}{|l|}{ Diaporthaceae } \\
\hline Diaporthe neotheicola & ColPat-448 & White beige & No & Irregular & White beige & No \\
\hline Diaporthe rhusicola & ColPat-444 & Light brown to white beige & No & Irregular & Light brown to white beige & Yes \\
\hline Phomopsis amygdali & ColPat-533 & Light brown to white beige & No & Irregular & Light to dark brown & No \\
\hline \multicolumn{7}{|l|}{ Valsaceae } \\
\hline Cytospora sp. & ColPat-465 & White beige to blue-olive gray & No & Irregular & Dark gray to olive green & No \\
\hline
\end{tabular}

${ }^{\mathrm{z}}$ Single conidial cultures were grown on PDA for 7 days at $25 \pm 2^{\circ} \mathrm{C}$ in darkness, and colony color was determined by means of a color scale (Kornerup and Wanscher 1963). 
mycelium with colors varying from white-gray to dark gray or olive green on PDA (Table 3; Fig. 2A to F). With age (from 10 to 14 days of incubation), all Botryosphaeriaceae isolates developed single or grouped, dark-brown to black-dark, ostiolate, globose, erumpent pycnidia, varying in diameter from 286.3 to $769.4 \mu \mathrm{m}$, on the surface of pistachio leaves on LA. When conidia from pycnidia were observed, Botryosphaeriaceae isolates were classified at the genus level resulting in the following four subgroups: (i) Botryosphaeria: aseptate, fusiform elliptical, hyaline conidia; (ii) Diplodia: aseptate, rounded, brown conidia; (iii) Dothiorella: (zero-) to one-septate, ovoid, hyaline becoming dark brown conidia; and (iv) Neofusicoccum: aseptate, fusoid-ellipsoidal (fusiform), hyaline conidia (Table 4). The second group was classified as Diaporthe because the colonies showed white, cottony, slow-growing, and raised mycelium developing prominent growth rings with irregular margins that became dark brown with age (Table 3; Fig. 2G to I). All isolates of the second group developed pycnidia over time on LA as well as on PDA, showing a characteristic white-beige-colored conidial mass on the pycnidia. Fruiting bodies were dark, ostiolate, globose, erumpent, and velvety and varied in diameter (216.1 to $354.9 \mu \mathrm{m})$. Two types of hyaline conidia were observed: alpha conidia $(\alpha)$ : small, oblong, slightly fusiform, and ligulated conidia; and beta conidia $(\beta)$ : filiform, smooth, slightly curved conidia (Table 4). Finally, a third group was composed of only one type of colony that developed white-beige to blue-olive-gray, flat, uniform mycelium with irregular colony margins (Table 3; Fig. 2J). Pycnidia were formed on LA after 14 days of incubation, and they were dark, erumpent, ostiolate, tuberculate, globous, velvet, and $485.2 \mu \mathrm{m}$ in diameter. Conidia were aseptate, elongate-allantoid, smooth-walled and hyaline (Table 4). This group of colonies was identified morphologically as Cytospora sp.

Effect of temperature on mycelial growth. All isolates used in this experiment were able to grow on PDA from 10 to $30^{\circ} \mathrm{C}$, showing little or sometimes a lack of mycelial growth development at both extreme temperatures tested $\left(5\right.$ and $\left.35^{\circ} \mathrm{C}\right)$. In general, different growth patterns were observed between the three fungal groups, and significant differences in optimum growth temperature and MGR $(P<$ 0.0001 in any cases) were observed between isolates. Optimum growth temperatures ranged from 19.5 to $27.7^{\circ} \mathrm{C}$, with the Cytospora sp. isolate ColPat-465 having the lowest growth temperature and the B. dothidea isolate ColPat-443 having the highest, and MGR ranged from 17.0 to $2.4 \mathrm{~mm} /$ day, with the $N$. parvum isolate ColPat-554 having the lowest MGR and the Dia. neotheicola isolate ColPat-448 having the highest. Among Botryosphaeriaceae isolates, the $B$. dothidea isolate ColPat-443 had the highest optimum growth temperature $\left(27.7^{\circ} \mathrm{C}\right)$, followed by the D. seriata isolate ColPat-557 $\left(26.6^{\circ} \mathrm{C}\right)$. Isolates belonging to the Dothiorella genus had the lowest optimum growth temperatures among the Botryosphaeriaceae isolates (19.6 and $22.0^{\circ} \mathrm{C}$ for the Dot. sarmentorum isolate ColPat-471 and Dothiorella sp. ColPat-470, respectively). The N. parvum isolate ColPat-554 showed the highest MGR value $(17.0 \mathrm{~mm} /$ day $)$, whereas $N$. mediterraneum showed the lowest MGR value $(5.1 \mathrm{~mm} /$ day $)$ among Botryosphaeriaceae isolates. Concerning Diaporthe species, the optimum growth temperature ranged from 23.2 to $26.6^{\circ} \mathrm{C}$, with the Dia. rhusicola isolate ColPat-444 having the lowest growth temperature and the Dia. neotheicola isolate ColPat-448 having the highest; MGR ranged from 2.4 to $6.4 \mathrm{~mm} / \mathrm{day}$, with the Dia. neotheicola isolate ColPat- 448 having the highest MGR and the Ph. amygdali isolate ColPat-533 having the lowest. Finally, the Cytospora sp. isolate ColPat-465 showed the lowest optimum growth temperature $\left(19.5^{\circ} \mathrm{C}\right)$ of the entire experimental group with an MGR of $4.3 \mathrm{~mm} /$ day (Table 5; Fig. 3; for interpretation of the results of this experiment with respect to the species name of each isolate, the reader is referred to the following section of this article).

Molecular characterization. Botryosphaeriaceae isolates were inferred by analyzing the combined alignment of the ITS, EF, and TUB sequences. This analysis contained 47 taxa, including the outgroup Neo. dimidiatum isolate CBS 499.66. The gene boundaries were as follows: ITS, 1 to 511 ; EF, 512 to 779 ; and TUB, 780 to 1,122 . A total of 1,122 characters, including gaps, were processed, of which 305 characters were parsimony informative and 788 were conserved sites. All positions containing gaps and missing data were eliminated when the MP analysis was performed. The MP analysis showed eight most parsimonious trees $(\mathrm{TL}=327$; $\mathrm{CI}=0.757$;
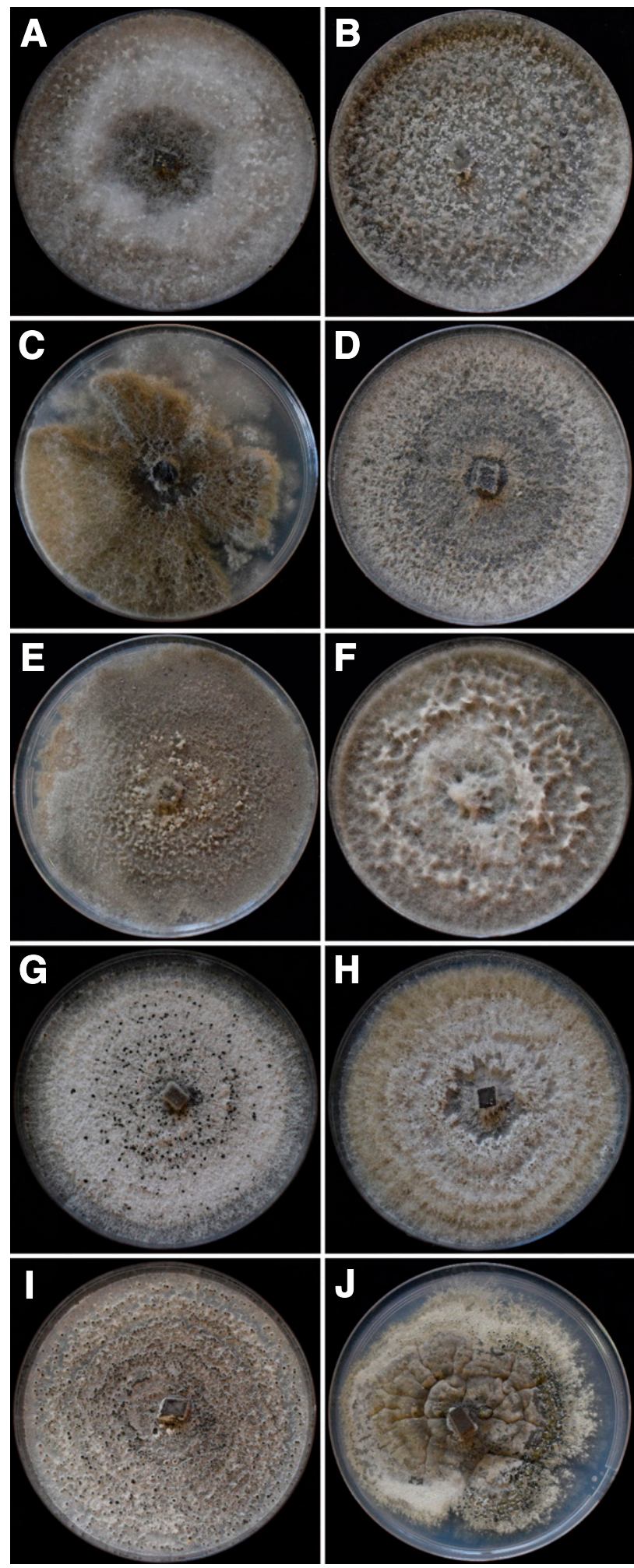

Fig. 2. Two-week-old colonies of the different fungal species isolated from English walnut in southern Spain growing on PDA at $25^{\circ} \mathrm{C}$ in darkness. Botryosphaeriaceae isolates: A, Botryosphaeria dothidea isolate ColPat-443; B, Diplodia seriata isolate ColPat-557; C, Dothiorella sarmentorum isolate ColPat-471; D, Dothiorella sp. isolate ColPat-470; E, Neofusicoccum mediterraneum isolate ColPat-556; and F, Neofusicoccum parvum isolate ColPat-554. Diaporthe isolates: G, Diaporthe neotheicola isolate ColPat-448; $\mathbf{H}$, Diaporthe rhusicola isolate ColPat-444; and I, Phomopsis amygdali isolate ColPat-533. J, Cytospora sp. isolate ColPat-465. 
$\mathrm{RI}=0.962 ; \mathrm{HI}=0.243 ; \mathrm{RC}=0.728$ ), of which one is shown in Figure 4. For BI analysis, the $\mathrm{K} 2+\mathrm{G}$ and $\mathrm{T} 92+\mathrm{G}$ models were used for the ITS region and for the EF and TUB genes, respectively. The tree obtained by BI analysis confirmed the topology obtained with MP. In this phylogenetic tree, the combined sequences of the Botryosphaeriaceae isolates clustered in six well-supported clades: (i) four isolates (ColPat-443, ColPat-446, ColPat-530, and ColPat-553) clustered together with reference GenBank sequences of B. dothidea (bootstrap support [BS; \%]/Bayesian posterior probability [PP]: 100/1.00); (ii) three isolates (ColPat-366, ColPat-367, and ColPat-557) clustered together with reference GenBank sequences of D. seriata (BS/PP: 99/ 1.00); (iii) three isolates (ColPat-370, ColPat-371, and ColPat-470) clustered together with reference GenBank sequences of Dot. omnivora and Diplodia juglandis (BS/PP: 97/1.00), considering our isolates as Dothiorella sp.; (iv) two isolates (ColPat-469 and ColPat-471) clustered together with reference GenBank sequences of Dot. sarmentorum (BS/PP: 93/0.97); (v) four isolates (ColPat-368, ColPat-369, ColPat-556, and ColPat-561) clustered together with reference GenBank sequences of N. mediterraneum (BS/PP: 100/1.00); and (vi) seven isolates (ColPat-447, ColPat-526, ColPat-528, ColPat-554, ColPat-558, ColPat-562, and ColPat-563) clustered together with reference GenBank sequences of $N$. parvum (BS/PP: 100/1.00).

With respect to the Diaporthe isolates, they were inferred by analyzing a combined alignment of the TUB and EF sequences. This analysis contained 34 taxa, including the outgroup Diaporthella corylina isolate CBS 121124. The gene boundaries were as follows: TUB, 1 to 481 ; and EF, 472 to 819 . A total of 819 characters including gaps were processed, of which 329 characters were parsimony informative and 275 were conserved sites. All positions containing gaps and missing data were eliminated when the MP analysis was performed. The MP analysis showed four most parsimonious trees $(\mathrm{TL}=724 ; \mathrm{CI}=0.722 ; \mathrm{RI}=0.805 ; \mathrm{HI}=0.278 ; \mathrm{RC}=0.581)$, of which one is shown in Figure 5. For BI analysis, the HKY+G model was used for both EF and TUB genes. The tree obtained by BI analysis confirmed the topology obtained with MP. In this phylogenetic tree, the combined sequences of the Diaporthe isolates clustered in three well-supported clades: (i) one isolate (ColPat-533) clustered together with reference GenBank sequences of $P$. amygdali (BS/PP: 100/1.00);

Table 4. Morphological characteristics of conidia of the representative fungal isolates collected from English walnut showing branch dieback and shoot blight in southern Spain

\begin{tabular}{|c|c|c|c|c|c|c|}
\hline \multirow[b]{2}{*}{$\begin{array}{l}\text { Family/fungal } \\
\text { species }\end{array}$} & \multirow[b]{2}{*}{ Isolate } & \multicolumn{4}{|c|}{ Conidia } & \multirow{2}{*}{$\begin{array}{c}\text { Pycnidia } \\
\begin{array}{c}\text { Diameter } \\
(\mu \mathrm{m})^{\mathrm{z}}\end{array}\end{array}$} \\
\hline & & Length $\times$ width $(\mu \mathrm{m})^{\mathrm{x}}$ & $\begin{array}{l}\text { Length/ } \\
\text { width }^{y}\end{array}$ & Morphology & Color & \\
\hline \multicolumn{7}{|l|}{ Botryosphaeriaceae } \\
\hline $\begin{array}{l}\text { Botryosphaeria } \\
\text { dothidea }\end{array}$ & ColPat-443 & $(20.5-) 24.4(-29.2) \times(3.0-) 5.2(-7.2)$ & $4.9 \pm 0.22$ & $\begin{array}{l}\text { Aseptate, fusiform elliptical } \\
\text { with a subobtuse apex and } \\
\text { a truncate base bearing a } \\
\text { minute basal frill. }\end{array}$ & Hyaline & $556.1 \pm 26.2$ \\
\hline Diplodia seriata & ColPat-557 & $(18.0-) 21.5(-24.6) \times(7.4-) 9.9(-11.6)$ & $2.2 \pm 0.05$ & $\begin{array}{l}\text { Aseptate, inner surface of } \\
\text { wall finely roughened, } \\
\text { apex broadly rounded, } \\
\text { base truncate. }\end{array}$ & Brown & $347.1 \pm 13.0$ \\
\hline $\begin{array}{l}\text { Dothiorella } \\
\text { sarmentorum }\end{array}$ & ColPat-471 & $(16.9-) 19.1(-21.2) \times(7.1-) 9.0(-11.3)$ & $2.2 \pm 0.04$ & $\begin{array}{l}\text { (Zero-) to one-septate, } \\
\text { slightly constricted at } \\
\text { the septum, ovoid with } \\
\text { a broadly rounded apex } \\
\text { and truncate base, brown } \\
\text { walled. }\end{array}$ & $\begin{array}{l}\text { Hyaline, } \\
\text { becoming } \\
\text { pigmented } \\
\text { brown }\end{array}$ & $554.9 \pm 21.0$ \\
\hline Dothiorella sp. & ColPat-470 & $(13.7-) 17.3(-21.9) \times(5.3-) 7.5(-10.3)$ & $2.4 \pm 0.15$ & $\begin{array}{l}\text { One-septate, ovoid with a } \\
\text { broadly rounded apex } \\
\text { and truncate base, brown } \\
\text { walled. }\end{array}$ & $\begin{array}{l}\text { Hyaline, } \\
\text { becoming } \\
\text { dark brown }\end{array}$ & $346.3 \pm 22.2$ \\
\hline $\begin{array}{l}\text { Neofusicoccum } \\
\text { mediterraneum }\end{array}$ & ColPat-556 & $(13.7-) 18.4(-21.1) \times(4.3-) 5.8(-7.1)$ & $3.23 \pm 0.08$ & $\begin{array}{l}\text { Aseptate, smooth, thin-walled, } \\
\text { fusoid-ellipsoidal, widest in } \\
\text { the middle-upper third, apex } \\
\text { subobtuse, subtruncate base. }\end{array}$ & Hyaline & $286.3 \pm 17.9$ \\
\hline $\begin{array}{l}\text { Neofusicoccum } \\
\text { parvum }\end{array}$ & ColPat-554 & $(12.3-) 16.9(-23.5) \times(4.8-) 5.9(-7.2)$ & $2.9 \pm 0.02$ & $\begin{array}{l}\text { Aseptate, ellipsoid with } \\
\text { subobtuse apex and truncate } \\
\text { base, smooth thin-walled, } \\
\text { becoming darker before } \\
\text { germination. }\end{array}$ & Hyaline & $769.4 \pm 25.8$ \\
\hline \multicolumn{7}{|l|}{ Diaporthaceae } \\
\hline $\begin{array}{l}\text { Diaporthe } \\
\text { neotheicola }\end{array}$ & ColPat-448 & $(4.8-) 6.6(-8.7) \times(1.1-) 1.9(-2.9)$ & $3.7 \pm 0.15$ & $\begin{array}{l}\alpha \text {-Conidia: Aseptate, } \\
\text { subcylindrical to } \\
\text { fusoid-ellipsoidal, widest } \\
\text { in middle. }\end{array}$ & Hyaline & $354.9 \pm 15.7$ \\
\hline $\begin{array}{l}\text { Diaporthe } \\
\text { rhusicola }\end{array}$ & ColPat-444 & $(5.6-) 6.7(-8.1) \times(1.1-) 2.2(-3.0)$ & $3.2 \pm 0.15$ & $\begin{array}{l}\alpha \text {-Conidia: Aseptate, } \\
\text { subcylindrical to } \\
\text { fusoid-ellipsoidal, } \\
\text { widest in middle. }\end{array}$ & Hyaline & $287.5 \pm 10.4$ \\
\hline $\begin{array}{c}\text { Phomopsis } \\
\text { amygdali }\end{array}$ & ColPat-533 & $(3.7-) 5.6(-7.3) \times(1.1-) 1.8(-2.9)$ & $3.3 \pm 0.15$ & $\begin{array}{l}\alpha \text {-Conidia: Aseptate, } \\
\text { subcylindrical to } \\
\text { fusoid-ellipsoidal, } \\
\text { widest in middle. }\end{array}$ & Hyaline & $216.1 \pm 13.7$ \\
\hline \multicolumn{7}{|l|}{ Valsaceae } \\
\hline Cytospora sp. & ColPat-465 & $(3.1-) 3.9(-4.6) \times(0.7-) 1.1(-1.6)$ & $3.7 \pm 0.16$ & $\begin{array}{l}\text { Aseptate, elongate-allantoid, } \\
\text { smooth-walled. }\end{array}$ & Hyaline & $485.2 \pm 19.8$ \\
\hline
\end{tabular}

\footnotetext{
${ }^{x}$ Mean and range values: length $\times$ width $(\mu \mathrm{m})$. The extremes of the conidial measurements are shown inside parenthesis. Values represent the mean of 30 conidia. y Conidia length/width ratio. Values represent the mean of 30 conidia \pm standard error of the mean.

${ }^{\mathrm{z}}$ Values represent the mean of the diameter $(\mu \mathrm{m})$ of 30 pycnidia \pm standard error of the mean.
} 
(ii) 10 isolates (ColPat-445, ColPat-448, ColPat-450, ColPat-451, ColPat-532, ColPat-551, ColPat-552, ColPat-555, ColPat-559, and ColPat-560) clustered together with reference GenBank sequences of Dia. neotheicola (BS/PP: 99/1.00); and (iii) one isolate (ColPat444) clustered together with reference GenBank sequences of Dia. rhusicola (BS/PP: 99/1.00). Additionally, one of our isolates (ColPat449) clustered into this later cluster close to reference GenBank sequences of Dia. chamaeropis and Dia. rhusicola (BS/PP: 88/0.99), and we considered this isolate to be Diaporthe sp.

Finally, the ITS, EF, and TUB sequences of the Cytospora spp. isolates ColPat-465, ColPat-633, and ColPat-634 were identified by performing a GenBank BLAST search. The ITS, EF, and TUB sequences of the isolates ColPat-465, ColPat-633, and ColPat-634 were incongruent when they were BLAST searched in GenBank. The BLAST searches showed a broad diversity of Cytospora species such as Cytospora abietis, C. carbonacea, C. cotini, C. rhodophila, C. prunicola, C. ribis, or Cytospora sp. with a similar percentage of identity (from 95 to $99 \%$; data not shown).

Pathogenicity tests. Inoculation of detached shoots. All isolates tested were able to induce lesions and internal wood discoloration on the inoculated walnut shoots at 3 weeks after inoculation in laboratory conditions (Fig. 6A). Significant differences in aggressiveness $(P<0.0001)$ were observed within species, with the mean lesion length ranging from 3.6 to $134.3 \mathrm{~mm}$, with the Cytospora sp. isolate ColPat-465 inducing the shortest lesion length and the $N$. parvum isolate ColPat-554 inducing the largest. The $N$. parvum isolate ColPat-554 was the most aggressive (lesion length $=134.3 \mathrm{~mm}$ ), followed by the $B$. dothidea isolate ColPat-443 (lesion length = $130.7 \mathrm{~mm}$ ) and the $N$. mediterraneum isolate ColPat-556 (lesion length $=112.5 \mathrm{~mm})$. The Dot. sarmentorum isolate ColPat-471 was the least aggressive within Botryosphaeriaceae isolates (lesion length $=54.6 \mathrm{~mm}$ ). With respect to Diaporthe fungi, the Dia. neotheicola isolate ColPat-448 showed the largest lesions (lesion length $=$ $23.3 \mathrm{~mm}$ ), followed by the $P h$. amygdali isolate ColPat-533 (lesion length $=17.6 \mathrm{~mm}$ ). Finally, the Cytospora sp. isolate ColPat-465 was the least aggressive among all the species in the entire experiment (lesion length $=3.6 \mathrm{~mm}$ ) (Fig. 7A). All fungal species were consistently reisolated from the margin of the necrotic lesion, with a percentage of reisolation ranging from 81.0 to $100 \%$. No positive reisolations were obtained from control shoots (Table 6). A significant negative linear correlation was observed between lesion length $(\mathrm{mm})$ and diameter $(\mathrm{mm})(r=-0.6747 ; P=0.0323)$.

Inoculation of attached shoots under field conditions. All isolates tested were able to induce lesions and internal wood discoloration on the inoculated walnut shoots under field conditions at 3 months after inoculation (Fig. 6B). Occasionally, serious attacks of branch dieback occurring in dry and dead branches were observed in shoots inoculated with mycelial plugs of the $N$. parvum isolate ColPat-554 (67\% of dead shoots), the D. seriata isolate ColPat-557 (25\% of dead shoots), and the $N$. mediterraneum isolate ColPat-556 (13\% of dead shoots) (Fig. 6C). Significant differences $(P<0.0001)$ in aggressiveness were observed within species, with the mean lesion length ranging from 5.1 to $90.5 \mathrm{~mm}$, with the Cytospora sp. isolate ColPat-465 inducing the shortest lesion length and the $N$. parvum isolate ColPat554 inducing the longest. The $N$. parvum isolate ColPat-554 was also the most aggressive (lesion length $=90.5 \mathrm{~mm}$ ), followed by the $N$. mediterraneum isolate ColPat-556 (lesion length $=28.0 \mathrm{~mm}$ ). The $P h$. amygdali isolate ColPat-533 showed similar aggressiveness (lesion length $=13.0 \mathrm{~mm}$ ) to that observed for the Botryosphaeriaceae species D. seriata isolate ColPat-557 (lesion length $=12.5 \mathrm{~mm}$ ) and B. dothidea isolate ColPat-443 (lesion length $=10.9 \mathrm{~mm}$ ). Finally, the Cytospora sp. isolate ColPat-465 was again the least aggressive among all the species in the entire experiment (lesion length $=$ $5.1 \mathrm{~mm}$ ) (Fig. 7B). Botryosphaeriaceae were the most consistent fungi reisolated from the inoculated shoots under field conditions, with $N$. parvum showing the highest percentage of reisolation (95.2\%). Diaporthe species were reisolated at a lower percentage ( 23.8 to $42.9 \%$ ) than Botryosphaeriaceae fungi (54.8 to $95.2 \%$ ). Cytospora sp. showed the lowest percentage of reisolation $(9.5 \%)$ among all species in the entire experiment. When reisolations were performed at $2 \mathrm{~cm}$ beyond the margin of the necrotic lesions, only $N$. parvum was reisolated at a low percentage $(2.4 \%)$. No positive reisolations were obtained from any evaluated tissue from control shoots (Table 6). Linear correlation analyses showed that there was no significant correlation $(r=-0.2845 ; P=0.4256)$ between lesion length $(\mathrm{mm})$ and branch diameter $(\mathrm{mm})$. However, a highly significant positive linear correlation $(r=0.8144 ; P=0.0041)$ was observed between lesion length $(\mathrm{mm})$ data from infected shoots evaluated in both laboratory and field conditions for the 10 fungal isolates tested.

Inoculation of detached walnut fruit. Dark-brown circular necrotic lesions varying in diameter among isolates tested were observed in the epicarp of the inoculated fruit at 3 weeks after inoculation in laboratory conditions. Internal discoloration and consistent fruit rot were observed in most of the inoculated fruit (Fig. 6D). In general, the Botryosphaeriaceae isolates were more aggressive with respect to the Diaporthe and Cytospora fungi. Significant differences in RAUDPC were observed within species $(P<0.0001)$. The $N$. parvum isolate ColPat-554 was the most aggressive (RAUDPC $=98.8 \%$ ), followed by the $B$. dothidea isolate ColPat-443 (RAUDPC $=65.3 \%$ ) and the $N$. mediterraneum isolate ColPat-556 (RAUDPC $=56.7 \%$ ). In

Table 5. Effect of temperature on mycelial growth of the representative fungal isolates selected in this study grown on PDA at $5,10,15,20,25,30$, and $35^{\circ} \mathrm{C}$ in darkness from 3 to 7 days $^{v}$

\begin{tabular}{|c|c|c|c|c|c|c|c|c|}
\hline \multirow[b]{2}{*}{ Family/fungal species } & \multirow[b]{2}{*}{ Isolate } & \multicolumn{3}{|c|}{ Analytics Beta modelw } & \multicolumn{3}{|c|}{ Temperature $\left({ }^{\circ} \mathbf{C}\right)^{\mathbf{x}, \mathbf{y}}$} & \multirow[b]{2}{*}{$\operatorname{MGR}(\mathbf{m m} / \text { day })^{\mathbf{y}, \mathbf{z}}$} \\
\hline & & $R^{2}$ & $a$ & $b$ & Optimum & Minimum & Maximum & \\
\hline \multicolumn{9}{|l|}{ Botryosphaeriaceae } \\
\hline Botryosphaeria dothidea & ColPat-443 & 0.9888 & 3.68 & 1.84 & $27.7 \mathrm{a}$ & 10.0 & 36.5 & $15.4 \mathrm{~b}$ \\
\hline Diplodia seriata & ColPat-557 & 0.9737 & 3.60 & 1.41 & $26.6 \mathrm{~b}$ & 4.0 & 35.5 & $13.4 \mathrm{c}$ \\
\hline Dothiorella sarmentorum & ColPat-471 & 0.9561 & 2.25 & 2.48 & $19.6 \mathrm{e}$ & 4.5 & 36.5 & 5.5 ef \\
\hline Dothiorella sp. & ColPat-470 & 0.9112 & 1.21 & 0.70 & $22.0 \mathrm{~d}$ & 9.0 & 30.5 & $9.0 \mathrm{~d}$ \\
\hline Neofusicoccum mediterraneum & ColPat-556 & 0.9454 & 1.47 & 2.43 & $23.7 \mathrm{c}$ & 5.0 & 35.0 & $5.1 \mathrm{fg}$ \\
\hline Neofusicoccum parvum & ColPat-554 & 0.9924 & 3.37 & 1.59 & $26.0 \mathrm{~b}$ & 5.0 & 36.0 & $17.0 \mathrm{a}$ \\
\hline \multicolumn{9}{|l|}{ Diaporthaceae } \\
\hline Diaporthe neotheicola & ColPat-448 & 0.9435 & 4.66 & 2.40 & $26.6 \mathrm{~b}$ & 10.0 & 35.0 & $2.4 \mathrm{~h}$ \\
\hline Diaporthe rhusicola & ColPat-444 & 0.8579 & 3.79 & 2.40 & $23.2 \mathrm{c}$ & 4.5 & 35.0 & $4.2 \mathrm{~g}$ \\
\hline Phomopsis amygdali & ColPat-533 & 0.9942 & 4.57 & 1.32 & $26.3 \mathrm{~b}$ & 5.0 & 32.5 & $6.4 \mathrm{~b}$ \\
\hline \multicolumn{9}{|l|}{ Valsaceae } \\
\hline Cytospora sp. & ColPat-465 & 0.9878 & 1.24 & 1.42 & $19.5 \mathrm{e}$ & 10 & 30.5 & $4.3 \mathrm{fg}$ \\
\hline
\end{tabular}

$\checkmark$ Data represents the average of four replicated Petri dishes per isolate and temperature combination.

w Analytis Beta model, where $R^{2}=$ coefficient of determination, and $a$ and $b=$ coefficients of regression.

${ }^{x}$ For each isolate, temperature average growth rates were adjusted to a regression curve to estimate the optimum growth temperature.

y Means in a column followed by the same letter do not differ significantly according to Tukey's HSD test at $P=0.05$ (Steel and Torrie 1985).

z MGR $=$ maximum growth rate $(\mathrm{mm} /$ day $)$ obtained by the Analytics Beta model at the optimum growth temperature. 
contrast, the remaining species showed similar effects on fruit infection, with RAUDPC values ranging from 34.0 to $39.8 \%$, with the Cytospora sp. isolate ColPat-465 having the lowest RAUDPC value and the Ph. amygdali isolate ColPat-533 having the highest (Fig. 8). Most of the fungal species were consistently reisolated from the margin of the necrotic lesion (93.0 to 100\%), except for Dot. sarmentorum and Cytospora sp. which showed 50\% reisolation. When reisolations were performed from the peduncle of the inoculated fruit, only some Botryosphaeriaceae species were reisolated (50.0 to 93.0\%), with $N$. parvum being the most consistently reisolated species. No positive reisolations were obtained from any evaluated tissue from control fruit (Table 6). A significant positive linear correlation was observed between RAUDPC (\%) and lesion length $(\mathrm{mm})$ data from the detached fruit and shoots inoculated in laboratory conditions $(r=0.8424$;

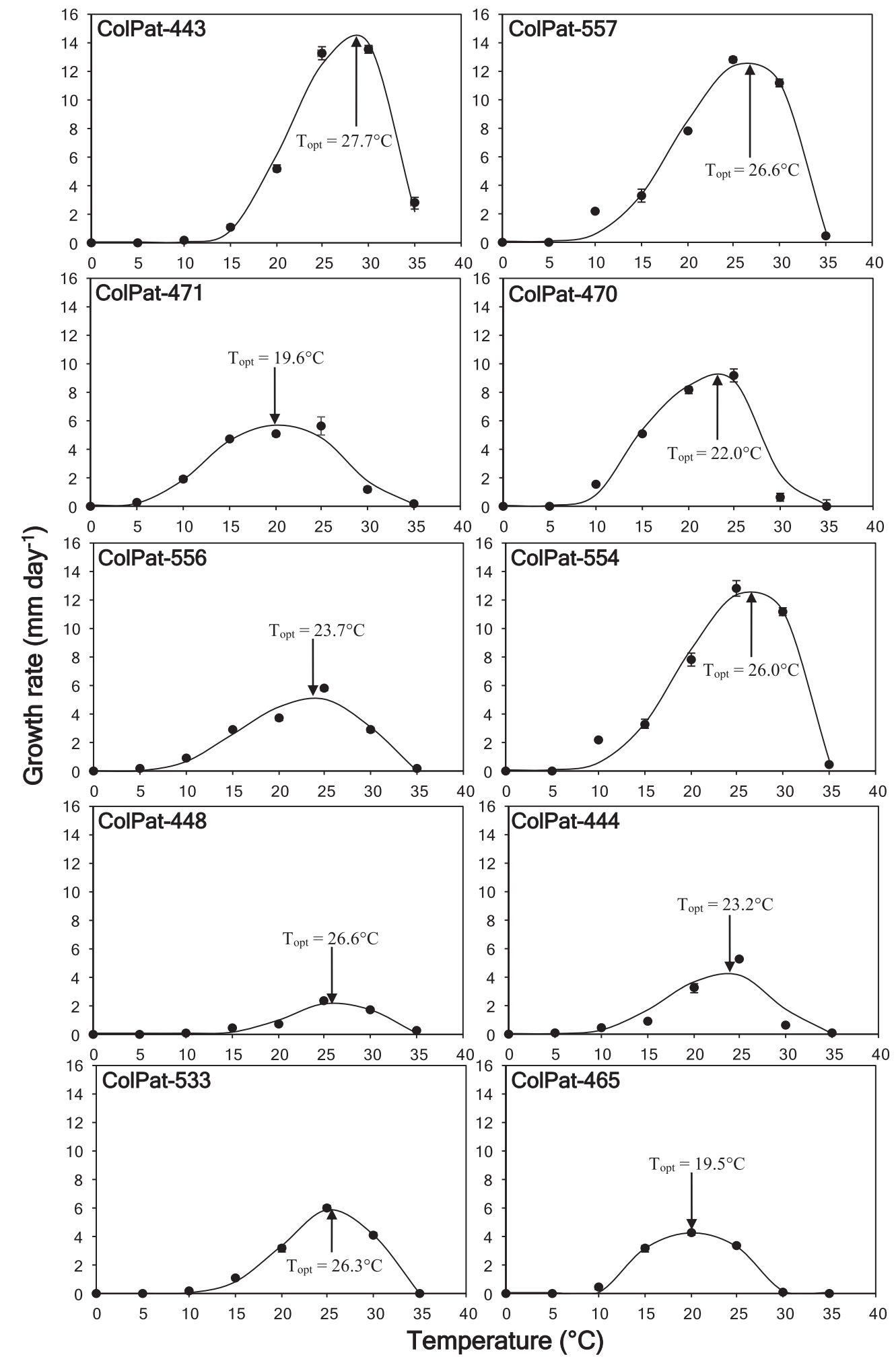

Fig. 3. Effect of temperature on mycelial growth rate ( $\mathrm{mm} /$ day) of the 10 representative isolates selected in this study grown on PDA at $5,10,15,20,25,30$, and $35^{\circ} \mathrm{C}$ in darkness for 3 to 7 days. For each isolate, average growth rates over temperature were adjusted to a nonlinear regression curve using the Analytis Beta model. Data points are the means of two independent experiments of four replicated Petri dishes each. Vertical bars are the standard error of the means. 
$P=0.0022)$ and from the detached fruit and shoots inoculated under field conditions $(r=0.9780 ; P<0.0001)$.

\section{Discussion}

A total of 10 commercial English walnut orchards showing branch dieback and shoot blight symptoms were surveyed in this study across southern Spain. The symptoms observed included twig and leaf blight associated with shoot blight and wood canker formation with internal discoloration and fungal fruiting structures embedded in the bark of the affected tissues (Fig. 1). Owing to the growing concern for nut growers in this area because the incidence of the disease has increased in the last few years, this study aimed to elucidate the etiology of the disease to provide a consistent diagnosis. This work is the first report to identify the fungal species causing this complex disease of English walnut in Spain and Europe.

All isolates recovered from affected wood tissues were grouped into three fungal groups (Botryosphaeriaceae, Cytospora, and Diaporthe) according to their morphological characteristics with respect to those described for each family or genus (Dissanayake et al. 2016; Gomes et al. 2013; Norphanphoun et al. 2017; Phillips et al. 2008, 2013, 2018). Botryosphaeriaceae isolates were the most consistently isolated fungi, followed by Diaporthe isolates. These two groups of fungi were recovered from seven out of the 10 surveyed orchards. They cooccurred in five orchards showing coinfections in the affected branches. The distribution of the populations of Botryosphaeriaceae and Diaporthe isolates in the English walnut plantations in southern Spain seemed to be balanced for the two groups, even though Botryosphaeriaceae isolates were always isolated more consistently than Diaporthe isolates. These results differ

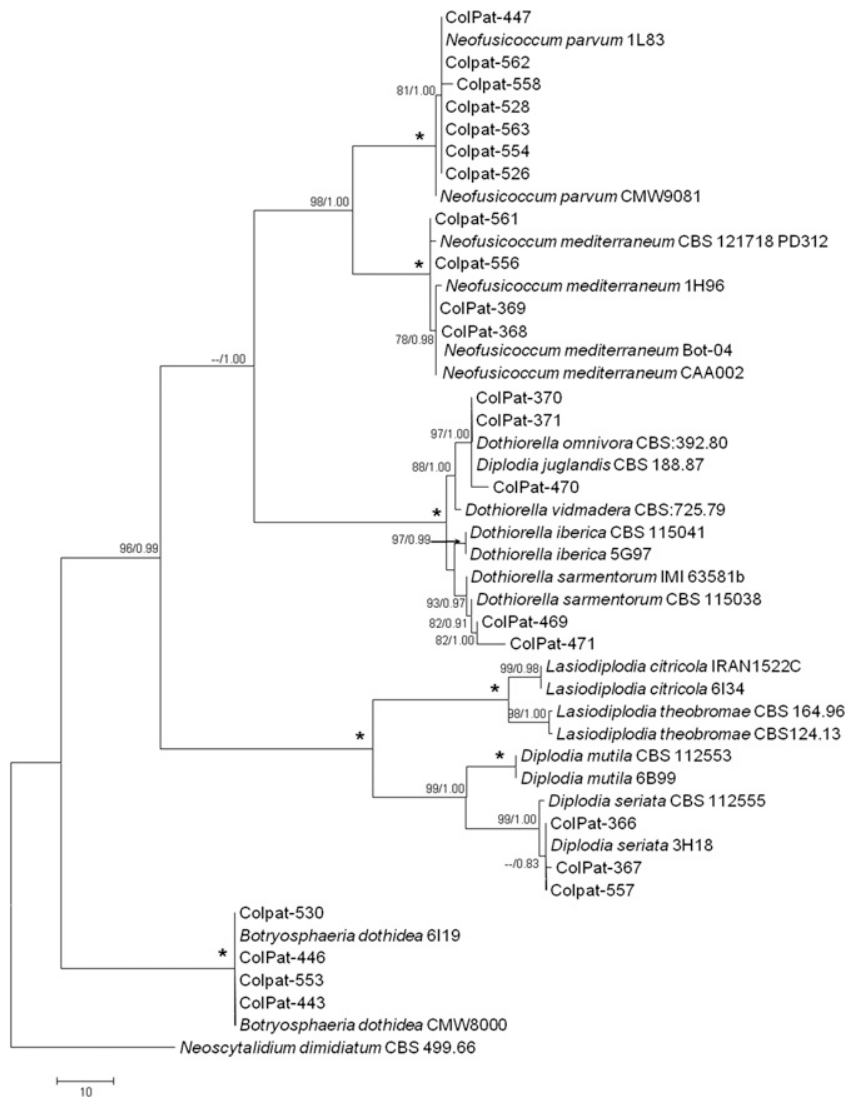

Fig. 4. One of eight most parsimonious trees (tree length $=327$; consistency index $=$ 0.757 ; retention index $=0.962$; homoplasy index $=0.243$; rescaled consistency index $=$ 0.728 ) obtained from a heuristic search of the combined internal transcribed spacer, elongation factor $1-\alpha$, and $\beta$-tubulin sequence alignment of fungal species belonging to Botryosphaeriaceae. Bootstrap support values (maximum parsimony [MP], $>70 \%$ ) and Bayesian posterior probabilities (PP, $>0.9$ ) are shown at the nodes (MP/PP). The asterisk $\left(^{*}\right)$ indicates full support (100/1.00). Neoscytalidium dimidiatum isolate CBS 499.66 was used as the outgroup. from those observed in California, where only $20 \%$ of the processed samples showed the cooccurrence of both Botryosphaeriaceae and Diaporthe fungi (Agustí-Brisach et al. 2019b; T. J. Michailides, unpublished data). In addition, Cytospora was recovered alone from a single orchard in our study, with minor relevance with respect to the other two fungal groups. Recently, this genus has also been associated with walnut canker disease in China (Fan et al. 2015; Zhao et al. 2018). Considering that Cytospora fungi caused the lowest incidence of disease, our results are in accordance with those obtained by Zhao et al. (2018), who observed this fungus only in a range from 5 to $10 \%$ of severely affected natural walnut forests.

The experiment evaluating the effect of temperature on mycelial growth showed a broad range of variation in the optimum growth temperature $\left(19.5\right.$ to $\left.27.7^{\circ} \mathrm{C}\right)$ between isolates. In general, Botryosphaeriaceae and Diaporthe isolates had high optimum growth temperatures of approximately 26 to $27^{\circ} \mathrm{C}$. Our results are in agreement with those obtained by several authors who showed similar ranges of temperature for these two groups of fungi in California and Spain. This information confirms that Botryosphaeriaceae and Diaporthe fungi are well adapted to the warm temperatures that occur in these locations, where canker diseases have been described as important diseases of woody crops (Agustí Brisach et al. 2019a, 2019b; Michailides and Hasey 2010; Moral et al. 2010, 2017, 2019). Moreover, Cytospora showed the lowest optimum growth temperature $\left(19.5^{\circ} \mathrm{C}\right)$ of all isolated in the entire experiment. This result is also in agreement with such obtained by Lawrence et al. (2017), who characterized Cytospora isolates from grapevines in North America. These authors observed that most of the Cytospora isolates showed slightly higher growth at 20 than at $25^{\circ} \mathrm{C}$.

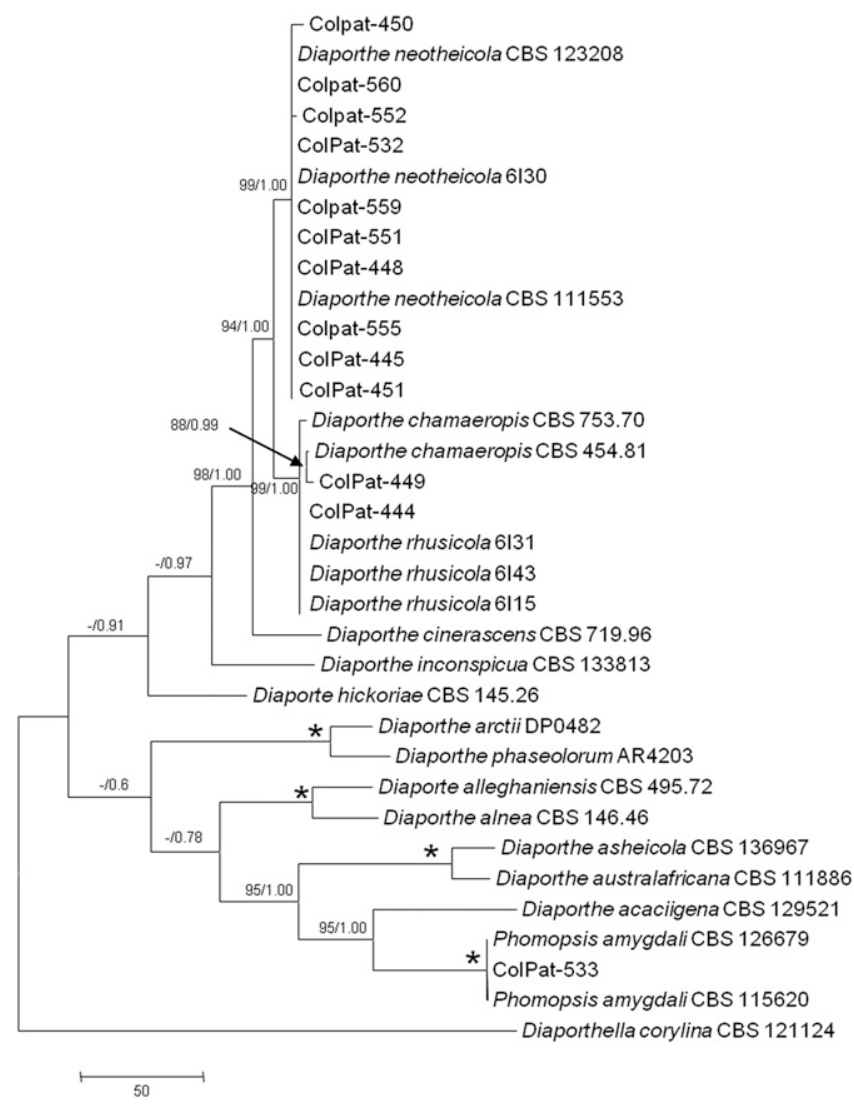

Fig. 5. One of four most parsimonious trees (tree length $=724$; consistency index $=$ 0.722 ; retention index $=0.805$; homoplasy index $=0.278$; rescaled consistency index $=$ 0.581) obtained from a heuristic search of the combined $\beta$-tubulin and elongation factor 1- $\alpha$ sequence alignment of Diaporthe fungi. Bootstrap support values (maximum parsimony [MP], $>70 \%$ ) and Bayesian posterior probabilities (PP, $>0.9$ ) are shown at the nodes (MP/PP). The asterisk $\left(^{*}\right)$ indicates full support (100/1.00). Diaporthella corylina isolate CBS 121124 was used as the outgroup. 
Although the phenotypic characteristics were useful for distinguishing our isolates between families and genera, sequencing and phylogenetic analysis were required to identify them at the species level. Based on previous studies that resolved the taxonomy of Botryosphaeriaceae (Chen et al. 2014; Dissanayake et al. 2016; Phillips et al. 2013, 2018), Cytospora (Norphanphoun et al. 2017), and Diaporthe (Chen et al. 2014; Gomes et al. 2013; Udayanga et al. 2014), sequences of the ITS, EF, and TUB gene regions were combined to identify our fungal isolates. Note that in the case of Diaporthe spp., the ITS region was not included
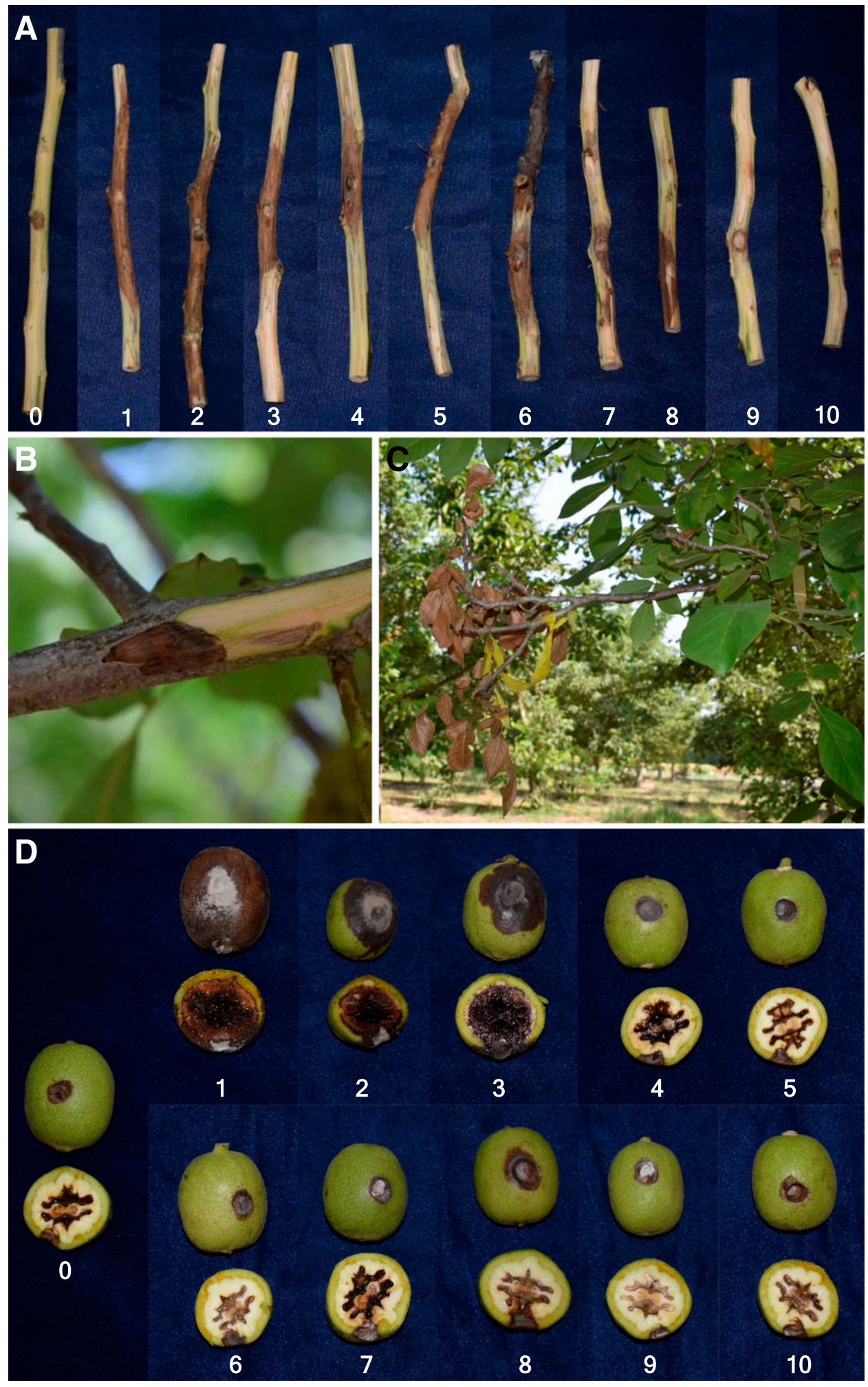

Fig. 6. A, Internal discoloration on detached shoots, and $\mathbf{D}$, external and internal lesions on green fruit of English walnut cv. Chandler at 3 weeks after inoculation with mycelial plugs of PDA ( $0=$ noninoculated control shoot) and of the 10 representative isolates selected for pathogenicity: $1=$ Neofusicoccum parvum isolate ColPat-554; $2=$ Botryosphaeria dothidea isolate ColPat-443; $3=\mathrm{N}$. mediterraneum isolate ColPat-556; $4=$ Diplodia seriata isolate ColPat-557; $5=$ Dot. sarmentorum isolate ColPat-471; $6=$ Dothiorella sp. isolate ColPat-470; 7 = Diaporthe neotheicola isolate ColPat-448; $8=$ Phomopsis amygdali isolate ColPat-533; 9 = Diaporthe rhusicola isolate ColPat-444; and 10 = Cytospora sp. isolate ColPat-465. B and C, internal discoloration and branch dieback, respectively, on shoots of English walnut cv. Chandler at 3 months after inoculation with mycelial plugs of the N. parvum isolate ColPat-554 under field conditions. 
in the alignment and phylogenetic analysis because previous studies on taxonomy into the genus Diaporthe showed a discordance of ITS versus other gene trees and a lack of informative morphological characters creating taxonomic ambiguities within this genus (Udayanga et al. 2014).
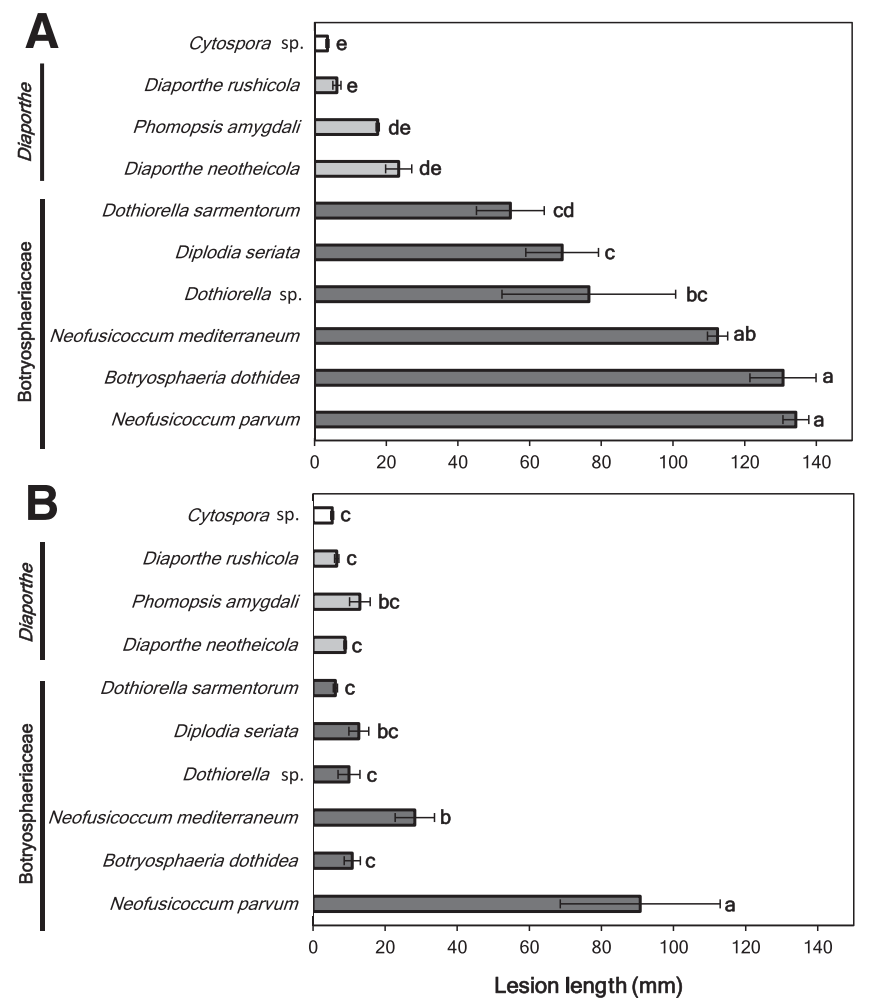

Fig. 7. Disease severity (lesion length, $\mathrm{mm}$ ) on $\mathbf{A}$, detached (laboratory conditions) and $\mathbf{B}$, attached (field conditions) shoots of English walnut cv. Chandler at 3 weeks and 4 months, respectively, after inoculation with mycelial plugs of representative fungal isolates belonging to the Botryosphaeriaceae (dark gray columns) and the Diaporthe (light gray columns) and Cytospora (white column) genera. For each isolate, columns represent the mean of six humidity chambers with five replicate shoots each in the artificial inoculations (A) and the mean of six walnut trees with two inoculated shoots in each in the field experiments (B). In each graph, columns with common letters do not differ significantly according to Tukey's HSD test $(\alpha=$ 0.05). Vertical lines on the columns are the standard error of the mean.
The following species were identified within the Botryosphaeriaceae group: B. dothidea, D. seriata, Dot. sarmentorum, Dothiorella sp., N. mediterraneum, and N. parvum. All of these species have been reported previously to cause branch dieback of walnut around the world (Chen et al. 2014; Li et al. 2015; Michailides and Hasey 2010), with the exception of Dot. sarmentorum, which we reported to be associated with this walnut disease for the first time. Concerning the Diaporthe group, most of our Diaporthe isolates were identified as Dia. neotheicola, making this species the most prevalent species in this group. The remaining isolates of this group were identified as Diaporthe sp. (ColPat-449), Dia. rhusicola (ColPat-444), and $P h$. amygdali (ColPat-533). Note that this latter has been traditionally described as the primary causal agent of cankers and wood decay in almond (Diogo et al. 2010; Gramaje et al. 2012; Rhouma et al. 2008). However, the detection of $P h$. amygdali as a causal agent of branch dieback in the walnut in this study confirms that this fungal species is not restricted to Prunus spp. (Gramaje et al. 2012). Indeed, $P h$. amygdali has also been isolated from other woody plants, such as grapevines in South Africa (Mostert et al. 2001) or Pieris japonica in the United States (Bienapfl and Balci 2013). Additionally, Dia. neotheicola (= Dia. foeniculina) has also been isolated from Foeniculum vulgare in Spain (Guarnaccia et al. 2018).

In both Botryosphaeriaceae and Diaporthe analyses, there was an isolate in each that was only identified at the genus level. The sequences of the isolates ColPat-370, ColPat-371, and ColPat-470 did not match any specific GenBank reference sequences of Botryosphaeriaceae with a high percentage of similarity $(\leq 96 \%)$ when they were BLAST searched in GenBank. When the combined alignment was conducted, these isolates were grouped together inside the Dothiorella sp. cluster, close to the reference sequences of D. juglandis CBS 188.87 (BS/PP: 97/1.0) and Dot. omnivora CBS 392.80 (BS/PP: 97/1.0). Owing to the unresolved ambiguity of this cluster, we considered these three isolates as Dothiorella sp. A similar situation was observed for the sequence of the isolate ColPat-449, belonging to the Diaporthe genus. The sequence of the ColPat-449 isolate did not match with any specific reference sequences of Diaporthe spp. with a high percentage of similarity $(\leq 96 \%)$ when BLAST searched in GenBank. Consequently, it clustered together with reference sequences of Dia. chamaeropis CBS 753.60 and CBS 454.81 (BS/PP: 88/0.99) in a subclade inside the main clade formed with reference sequences of Dia. rhusicola. These isolates did not match with any currently described species belonging to Botryosphaeriaceae or Diaporthe spp.; thus, owing to the great advances in fungal taxonomy that have occurred during the last few years, studies to determine their identity to describe them as potential

Table 6. Frequency of reisolation (\%) of fungal isolates used in the pathogenicity tests from the inoculated detached and attached shoots, and green fruits of English walnut

\begin{tabular}{|c|c|c|c|c|c|c|}
\hline \multirow[b]{3}{*}{ Family/fungal species } & \multirow[b]{3}{*}{ Isolate } & \multicolumn{5}{|c|}{ Frequency of reisolation $(\%)^{\mathrm{z}}$} \\
\hline & & \multirow{2}{*}{$\frac{\text { Detached shoots }}{\text { Margin }}$} & \multicolumn{2}{|c|}{ Attached shoots } & \multicolumn{2}{|c|}{ Fruit } \\
\hline & & & Margin & $2 \mathrm{~cm}$ beyond the margin & Margin & Peduncle \\
\hline \multicolumn{7}{|l|}{ Botryosphaeriaceae } \\
\hline Botryosphaeria dothidea & ColPat-443 & 100 & 66.6 & 0.0 & 100 & 86.0 \\
\hline Diplodia seriata & ColPat-557 & 88.0 & 90.5 & 0.0 & 100 & 71.5 \\
\hline Dothiorella sarmentorum & ColPat-471 & 100 & 59.5 & 0.0 & 50.0 & 0.0 \\
\hline Dothiorella sp. & ColPat-470 & 93.0 & 54.8 & 0.0 & 93.0 & 0.0 \\
\hline Neofusicoccum mediterraneum & ColPat-556 & 86.0 & 54.8 & 0.0 & 100 & 50.0 \\
\hline Neofusicoccum parvum & ColPat-554 & 100 & 95.2 & 2.4 & 100 & 93.0 \\
\hline \multicolumn{7}{|l|}{ Diaporthaceae } \\
\hline Diaporthe neotheicola & ColPat-448 & 100 & 42.9 & 0.0 & 100 & 0.0 \\
\hline Diaporthe rhusicola & ColPat-444 & 100 & 23.8 & 0.0 & 100 & 0.0 \\
\hline Phomopsis amygdali & ColPat-533 & 100 & 42.9 & 0.0 & 100 & 0.0 \\
\hline \multicolumn{7}{|l|}{ Valsaceae } \\
\hline Cytospora sp. & ColPat-465 & 81.0 & 9.5 & 0.0 & 50.0 & 0.0 \\
\hline Control & PDA & 0.0 & 0.0 & 0.0 & 0.0 & 0.0 \\
\hline
\end{tabular}


new species within the Dothiorella or Diaporthe genera should be conducted in the future.

With respect to our Cytospora isolates, their sequences matched many reference sequences of species belonging to the Cytospora genus with high similarity; consequently, we were not able to identify them at the species level. Owing to the low importance of this fungi in our study with respect to their frequency in field infections as well as

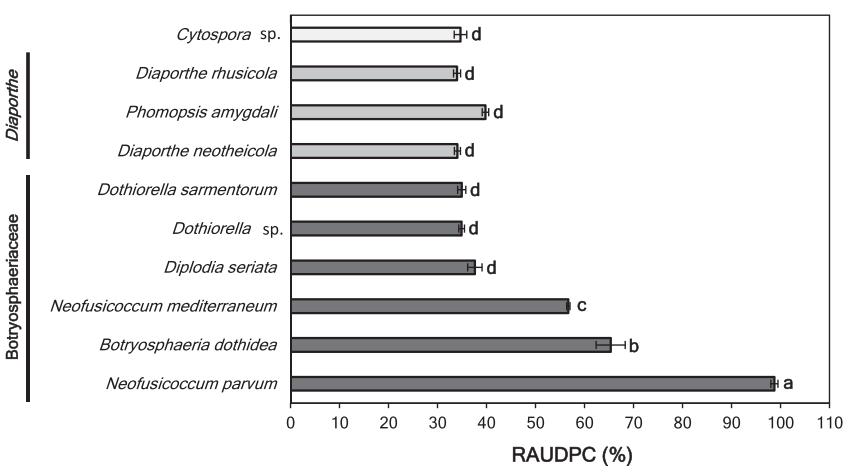

Fig. 8. Relative area under the disease progress curve (RAUDPC; \%) on green fruits (epicarp) of English walnut cv. Chandler obtained at 21 days after inoculation with mycelial plugs of representative isolates belonging to the Botryosphaeriaceae (dark gray columns) and the Diaporthe (light gray columns) and Cytospora (white column) genera. Columns represent the mean values of 30 replicate fruits. Columns with common letters do not differ significantly according to Tukey's HSD test $(\alpha=0.05)$. Vertical lines on the columns are the standard error of the mean. in aggressiveness (see below), further analyses to infer their identity were not conducted, and they were identified as Cytospora sp. As we indicated above, Cytospora species have also been associated with branch dieback and shoot blight in other walnut growing areas of the world, such as China, but with low importance (Fan et al. 2015; Zhao et al. 2018).

In general, a pathogenicity test revealed that Botryosphaeriaceae were the most aggressive fungi to walnut in all tissues evaluated (detached and attached shoots and fruit), followed by Diaporthe spp. and Cytospora sp.; $N$. parvum was the most aggressive among the remaining species tested in any tissues evaluated, followed by $B$. dothidea or N. mediterraneum; and D. seriata, Dot. sarmentorum, and Dothiorella sp. were the least aggressive species within the Botryosphaeriaceae group. Diaporthe were less aggressive than Botryosphaeriaceae species, and significant differences in aggressive between the Diaporthe isolates were observed depending on the infected tissue. All of our results are in accordance with those obtained by Chen et al. (2014) in California, where $N$. parvum was the most aggressive species to walnut after $L$. citricola. These same authors also described $N$. mediterraneum as highly aggressive and $D$. seriata, Dot. iberica, Dia. neotheicola, and Dia. rhusicola as slightly aggressive to walnut. Finally, Cytospora sp. was the least aggressive species, causing minimum cankers and internal discoloration in this study. Similar results were obtained by Moral et al. (2017), who tested $C$. pruinosa in olive by inoculating detached shoots and potted plants of olive. In this study, C. pruinosa was the least aggressive species in the detached shoots with respect to N. mediterraneum, Nothophoma quercina, Comoclathris incompta, and Diaporthe sp., and it was not pathogenic to potted olive plants. Finally, the

LATE SPRING-SUMMER

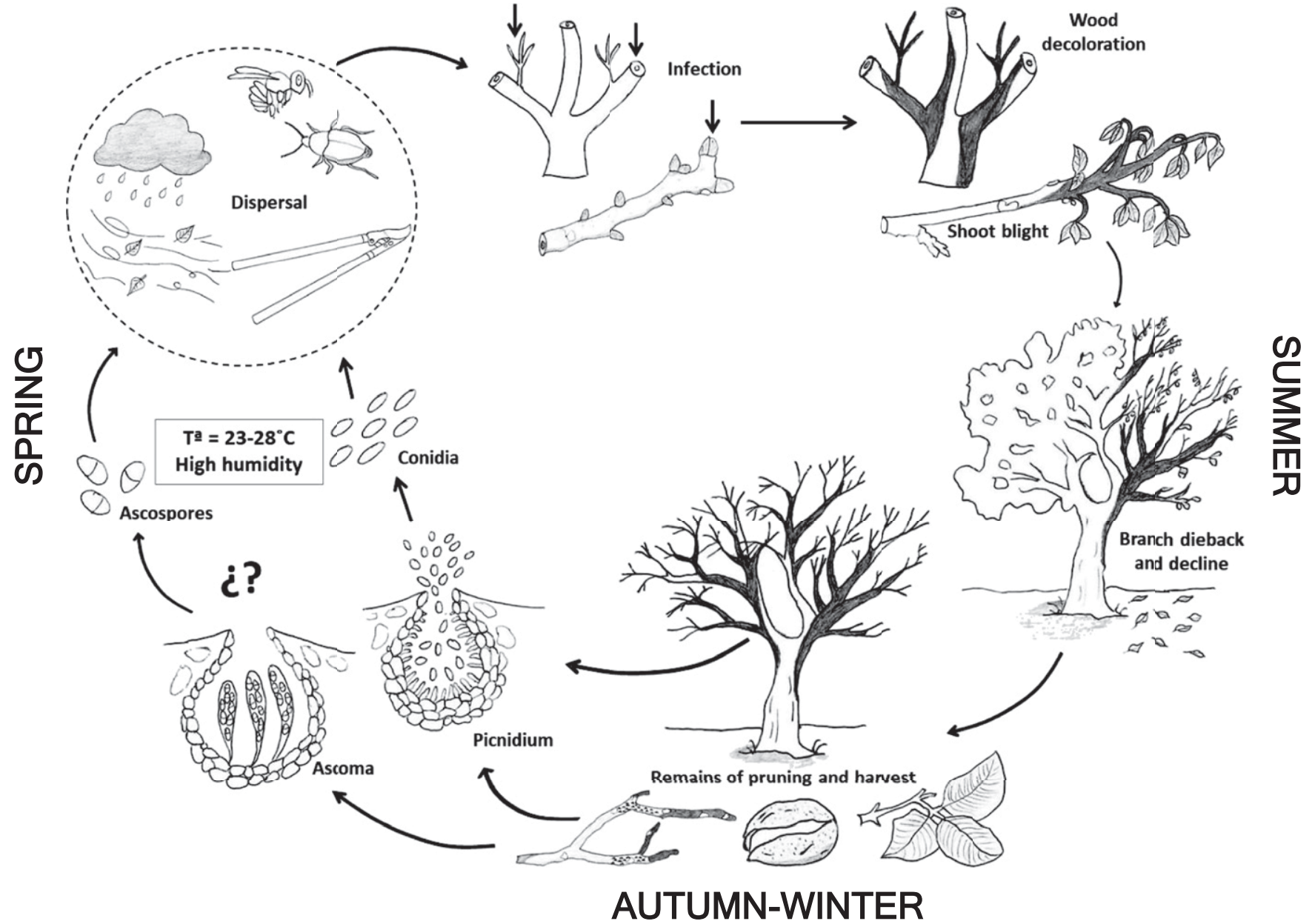

Fig. 9. The biological cycle of branch dieback and shoot blight of the English walnut adapted to the environmental conditions in southern Spain. 
Botryosphaeriaceae isolates were the most consistently reisolated fungi from all inoculated tissues in our study, with $N$. parvum showing the highest percentage of reisolation in any case. Note that the consistency of reisolation of the three groups of fungi was in accordance with respect to the aggressiveness of each isolate observed in the pathogenicity tests. This suggests that the most aggressive species (Botryosphaeriaceae) can survive after infection longer in infected tissues than less aggressive species, such as Diaporthe or Cytospora.

The results obtained in this study are relevant because we determined the main causal agents of branch dieback and shoot blight of English walnut in Spain. This study represents the first report of B. dothidea, Cytospora sp., D. seriata, Dia. neotheicola, Dia. rhusicola, Dot. sarmentorum, Dothiorella sp., N. mediterraneum, N. parvum, and Ph. amygdali causing branch dieback and shoot blight of English walnut in Spain and Europe. Altogether, these results are the first approach to describe the etiology of this complex disease, which is causing a growing concern in our walnut plantations. By understanding the diversity of the fungal species associated with the disease, we will be able to pursue studies on biology, epidemiology, and control to establish optimum management strategies to prevent infections. Special attention must be given to the infection process with respect to pruning wounds as well as the potential interaction between pathogens. Recently, studies conducted in California by Agustí-Brisach et al. (2019b) showed that both Botryosphaeriaceae and Diaporthe fungi infect walnut shoots through pruning wounds with higher severity when infections occur in the pith tissue instead of in the bark. Additionally, these same authors suggest that both groups of fungi could establish an antagonistic or synergistic interaction during the wood infection process when coinfections occur in the same tissue (Agustí-Brisach et al. 2019b). These later aspects should be considered for studies of the biology and epidemiology of the disease in Spanish plantations. The information generated in the present study has been helpful for obtaining the first description of the biological cycle of the disease according to the environmental conditions in southern Spain (Fig. 9). This region is characterized by a Mediterranean climate with a rainy spring with moderate temperatures followed by a long dry and hot period during the late spring and summer, which favors the dispersal and infection of trunk pathogens. This life cycle should be used to determine the critical moments with respect to the infection of the pathogens to carry out effective management strategies against the disease. Finally, our results are not enough to determine the distribution of Botryosphaeriaceae and Diaporthe populations across Spain. A broad survey extended across the Iberian Peninsula should be conducted in the coming years to determine the fungal diversity of canker pathogens in walnut in Spain. It would be helpful to better understand this complex disease and to establish a possible correlation between fungal populations and the agronomical and environmental conditions of the different walnut growing areas of this country.

\section{Acknowledgments}

The authors thank Crisolar and Mañán OPFHs and the private companies Almendras Francisco Morales, Bain (Borges Group), and Todolivo for their collaboration

\section{Literature Cited}

Agustí-Brisach, C., López-Moral, A., Raya-Ortega, M. C., Franco, R., RocaCastillo, L. F., and Trapero, A. 2019a. Occurrence of grapevine trunk diseases affecting the native cultivar Pedro Ximénez in southern Spain. Eur. J. Plant Pathol. 153:599-625.

Agustí-Brisach, C., Moral, J., Felts, D., Trapero, A., and Michailides, T. J. $2019 \mathrm{~b}$. Interaction between Diaporthe rhusicola and Neofusicoccum mediterraneum causing branch dieback and fruit blight of English walnut in California, and effect of pruning wounds to the infection. Plant Dis. 103:1196-1205.

Agustí-Brisach, C., Moral, J., Pérez-Rodríguez, M., Roca, L. F., Raya-Ortega, M. C., Romero, J., and Trapero, A. 2017. Etiología y perspectivas de control de los chancros y desecaciones de ramas de olivo causados por hongos. Phytoma Esp. 292:54-60.

Analytical Software. 2013. Statistix10 User's Manual. Analytical Software, Tallahassee, FL.

Armengol, J., Vicent, A., Torné, L., García-Figueres, F., and García-Jiménez, J. 2001. Fungi associated with esca and grapevine declines in Spain: A threeyear survey. Phytopathol. Mediterr. 40:325-329.
Aroca, A., García-Figueres, F., Bracamonte, L., Luque, J., and Raposo, R. 2006. A survey of trunk pathogens within rootstocks of grapevines in Spain. Eur. J. Plant Pathol. 115:195-202.

Aroca, A., Raposo, R., Gramaje, D., Armengol, J., Martos, S., and Luque, J. 2008 First report of Lasiodiplodia theobromae associated with decline of grapevine rootstock mother plants in Spain. Plant Dis. 92:832.

Barnett, H. L., and Hunter, B. B. 1998. Illustrated Genera of Imperfect Fungi, 4th Ed. APS Press, St. Paul, MN.

Bienapfl, J. C., and Balci, Y. 2013. Phomopsis blight: A new disease of Pieris japonica caused by Phomopsis amygdali in the United States. Plant Dis. 97: 1403-1407.

Campbell, C. L., and Madden, L. V. 1990. Introduction to Plant Disease Epidemiology. Wiley, New York, NY

Carbone, I., and Kohn, L. M. 1999. A method for designing primer sets for speciation studies in filamentous ascomycetes. Mycologia 91:553-556.

Chen, S. F., Morgan, D. P., Hasey, J. K., Anderson, K., and Michailides, T. J. 2014 Phylogeny, morphology, distribution, and pathogenicity of Botryosphaeriaceae and Diaporthaceae from English walnut in California. Plant Dis. 98:636-652.

Daniels, D. A., Nix, K. A., Wadl, P. A., Vito, L. M., Wiggins, G. J., Windham, M. T., Ownley, B. H., Lambdin, P. L., Grant, J. F., Merten, P., Klingeman, W. E., and Hadziabdic, D. 2016. Thousand cankers disease complex: A forest health issue that threatens Juglans species across the U.S. Forests 7:260.

Dhingra, O. D., and Sinclair, J. B. 1995. Basic Plant Pathology Methods, 2nd Ed. CRC Press, Boca Raton, FL.

Díaz, G. A., Latorre, B. A., Ferrada, E., Gutiérrez, M., Bravo, F., and Lolas, M 2018. First report of Diplodia mutila causing branch dieback of English walnut cv. Chandler in the Maule Region, Chile. Plant Dis. 102:1451.

Diogo, E. L. F., Santos, J. M., and Phillips, A. J. L. 2010. Phylogeny, morphology and pathogenicity of Diaporthe and Phomopsis species on almond in Portugal Fungal Divers. 44:107-115.

Dissanayake, A. J., Phillips, A. J. L., Li, X. H., and Hyde, K. D. 2016 Botryosphaeriaceae: Current status of genera and species. Mycosphere 7 1001-1073.

Fan, X. L., Hyde, K. D., Liu, M., Liang, Y. M., and Tian, C. M. 2015. Cytospora species associated with walnut canker disease in China, with description of a new species $C$. gigalocus. Fungal Biol. 119:310-319.

Felsenstein, J. 1985. Confidence limits on phylogenies: An approach using the bootstrap. Evolution 39:783-791.

Glass, N. L., and Donaldson, G. 1995. Development of primer sets designed for use with PCR to amplify conserved genes from filamentous ascomycetes. Appl. Environ. Microbiol. 61:1323-1330.

Gomes, R. R., Glienke, C., Videira, S. I. R., Lombard, L., Groenewald, J. Z., and Crous, P. W. 2013. Diaporthe: A genus of endophytic, saprobic and plant pathogenic fungi. Persoonia 31:1-41.

Gramaje, D., Agustí-Brisach, C., Pérez-Sierra, A., Moralejo, E., Olmo, D., Moster, L., Damm, U., and Armengol, J. 2012. Fungal trunk pathogens associated with wood decay of almond trees on Mallorca (Spain). Persoonia 28:1-13.

Guarnaccia, V., Groenewald, J. Z., Woodhall, J., Armengol, J., Cinelli, T., Eichmeier, A., Ezra, D., Fontaine, F., Gramaje, D., Gutierrez-Aguirregabiria, A., Kaliterna, J., Kiss, L., Larignon, P., Luque, J., Mugnai, L., Naor, V., Raposo, R., Sándor, E., Váczy, K. Z., and Crous, P. W. 2018. Diaporthe diversity and pathogenicity revealed from a broad survey of grapevine diseases in Europe. Persoonia 40:135-153.

Hau, B., and Kranz, J. 1990. Mathematics and statistics for analyses in epidemiology. Pages 15-21 in: Epidemics of Plant Diseases. J. Kranz, ed. Springer-Verlag, Berlin, Germany.

International Nut and Dried Fruit Council. 2017. https://www.nutfruit.org/ industry/statistics

Kornerup, A., and Wanscher, J. H. 1963. Methuen Handbook of Colour. Methuen and Co., London, U.K.

Kumar, S., Stecher, G., and Tamura, K. 2016. MEGA7: Molecular Evolutionary Genetics Analysis version 7.0 for bigger datasets. Mol. Biol. Evol. 33: $1870-1874$

Lawrence, D. P., Travadon, R., Pouzoulet, J., Rolshausen, P. E., Wilcox, W. F., and Baumgartner, K. 2017. Characterization of Cytospora isolates from wood cankers of declining grapevine in North America, with the descriptions of two new Cytospora species. Plant Pathol. 66:713-725.

Li, G. Q., Liu, F. F., Li, J. Q., Liu, Q. L., and Chen, S. F. 2015. Characterization of Botryosphaeria dothidea and Lasiodiplodia pseudotheobromae from English walnut in China. J. Phytopathol. 164:348-353.

López-Larrinaga, F., Mellado-Bermejo, E., Valadares de Queirós, R., FiguerasLorenzo, M., and Gomes-Pires, J. 2017. Las nuevas plantaciones de nogal en España. Fruticultura 64:45-59.

López-Moral, A., Raya-Ortega, M. C., Agustí-Brisach, C., Roca, L. F., Lovera, M. Luque, F., Arquero, O., and Trapero, A. 2017. Morphological, pathogenic, and molecular characterization of Colletotrichum acutatum isolates causing almond anthracnose in Spain. Plant Dis. 101:2034-2045.

Lovera, M., Rodríguez, R. A., Arquero, O., and Trapero, A. 2017a. Lesiones necróticas en parte aérea de nogal I. Caracterización y etiología. Fruticultura 64:70-89.

Lovera, M., Rodríguez, R. A., Arquero, O., and Trapero, A. 2017b. Lesiones necróticas en parte aérea de nogal II. Factores que influyen en su incidencia y severidad. Fruticultura 64:90-99. 
Martin, M. T., and Cobos, R. 2007. Identification of fungi associated with grapevine decline in Castilla y León (Spain). Phytopathol. Mediterr. 46:18-25.

Melgarejo, P., García-Jiménez, J., Jordá, M. C., López, M. M., Andrés, M. F., and Duran-Vila, N. 2010. Patógenos de Plantas Descritos en España, 2nd Ed. Ministerio de Medio Ambiente y Medio Rural y Marino (MARM), Madrid, Spain.

Michailides, T. J., and Hasey, J. 2010. Botryosphaeria and Phomopsis cankers of walnuts in California. Walnut Husk Fly Field Meeting. University of California Cooperative Extension, Half Moon Bay, CA.

Ministerio de Agricultura, Pesca y Alimentación. 2017. https://www.mapa.gob.es/ es/estadistica/temas/estadisticas-agrarias/agricultura/superficies-produccionesanuales-cultivos/

Moral, J., Agustí-Brisach, C., Pérez Rodríguez, M., Xavíer, C., Raya, M. C., Rhouma, A., and Trapero, A. 2017. Identification of fungal species associated with branch dieback of olive and resistance of table cultivars to Neofusicoccum mediterraneum and Botryosphaeria dothidea. Plant Dis. 101: 306-316.

Moral, J., Morgan, D., Trapero, A., and Michailides, T. J. 2019. Ecology and epidemiology of diseases of nut crops and olives caused by Botryosphaeriaceae fungi in California and Spain. Plant Dis. 103:1809-1827.

Moral, J., Muñoz-Díez, C., González, N., Trapero, A., and Michailides, T. J. 2010. Characterization and pathogenicity of Botryosphaeriaceae species collected from olive and other hosts in Spain and California. Phytopathology 100: 1340-1351.

Mostert, L., Crous, P. W., Kang, J. C., and Phillips, A. J. L. 2001. Species of Phomopsis and a Libertella sp. occurring on grapevines with specific reference to South Africa: Morphological, cultural, molecular and pathological characterization. Mycologia 93:146-167.

Norphanphoun, C., Doilom, M., Daranagama, D. A., Phookamsak, R., Wen, T. C., Bulgakov, T. S., and Hyde, K. D. 2017. Revisiting the genus Cytospora and allied species. Mycosphere 8:51-97.

O'Donnell, K., and Cigelnik, E. 1997. Two divergent intragenomic rDNA ITS2 types within a monophyletic lineage of the fungus Fusarium are nonorthologous. Mol. Phylogenet. Evol. 7:103-116.

Olmo, D., Gramaje, D., and Armengol, J. 2017. Hongos asociados a las enfermedades de la madera del almendro en la Isla de Mallorca. Fruticultura 54:18-29.

Phillips, A. J. L., Alves, A., Abdollahzadeh, J., Slippers, B., Wingfield, M. J., Groenewald, J. Z., and Crous, P. W. 2013. The Botryosphaeriaceae: Genera and species known from culture. Stud. Mycol. 76:51-167.

Phillips, A. J. L., Alves, A., Pennycook, S. R., Johnston, P. R., Ramaley, A., Akulov, A., and Crous, P. W. 2008. Resolving the phylogenetic and taxonomic status of dark-spored teleomorph genera in the Botryosphaeriaceae. Persoonia 21:29-55.

Phillips, A. J. L., Hyde, K. D., Alves, A., and Liu, J. K. 2018. Families in Botryosphaeriales: A phylogenetic, morphological and evolutionary perspective. Fungal Divers. 94:1-22.
Rhouma, A., Triki, M. A., Ouerteni, K., and Mezghanni, M. 2008. Chemical and biological control of Phomopsis amygdali the causal agent of constriction canker of almond in Tunisia. TJPP 3:69-77.

Rolshausen, P. E., Úrbez-Torres, J. R., Rooney-Latham, S., Eskalen, A., Smith, R. J., and Gubler, W. D. 2010. Evaluation of pruning wound susceptibility and protection against fungi associated with grapevine trunk diseases. Am. J. Enol. Vitic. 61:113-119.

Ronquist, F., Teslenko, M., Van Der Mark, P., Ayres, D. L., Darling, A., Höhna S., Larget, B., Liu, L., Suchard, M. A., and Huelsenbeck, J. P. 2012. MrBayes 3.2: Efficient Bayesian phylogenetic inference and model choice across a large model space. Syst. Biol. 61:539-542.

Sánchez, M. E., Venegas, J., and Romero, M. A. 2003. Botryosphaeria and related taxa causing oak canker in Southwestern Spain. Plant Dis. 87: 1515-1521.

Sánchez-Solana, J., Sánchez, M. E., Roca, L. F., Raya, M. C., Agustí-Brisach, C., Arquero, O., and Trapero, A. 2018. Enfermedades del castaño causadas por hongos en Andalucía. Fruticultura 64:22-43.

Steel, R. G. D., and Torrie, J. H. 1985. Bioestadística, 2nd Ed. McGraw-Hill, Bogotá, Colombia.

Swofford, D. L. 2003. PAUP* 4.0b10: Phylogenetic Analysis Using Parsimony (*and other methods). Sinauer Associates, Sunderland, Massachusetts.

Trouillas, F. P., Urbez-Torres, J. R., Peduto, F., and Gubler, W. D. 2010. First report of twig and branch dieback of English walnut (Juglans regia) caused by Neofusicoccum mediterraneum in California. Plant Dis. 94:1267.

Udayanga, D., Castlebury, L. A., Rossman, A. Y., Chukeatirote, E., and Hyde, K. D. 2014. Insights into the genus Diaporthe: Phylogenetic species delimitation in the $D$. eres species complex. Fungal Divers. 67:203-229.

Úrbez-Torres, J. R. 2011. The status of Botryosphaeriaceae species infecting grapevines. Phytopathol. Mediterr. 50:S5-S45.

Úrbez-Torres, J. R., and Gubler, W. D. 2009. Pathogenicity of Botryosphaeriaceae species isolated from grapevine cankers in California. Plant Dis. 93:584-592.

Úrbez-Torres, J. R., Leavitt, G. M., Voegel, T. M., and Gubler, W. D. 2006 Identification and distribution of Botryosphaeria spp. associated with grapevine cankers in California. Plant Dis. 90:1490-1503.

Úrbez-Torres, J. R., Peduto, F., Vossen, P. M., Krueger, W. H., and Gubler, W. D. 2013. Olive twig and branch dieback: Etiology, incidence, and distribution in California. Plant Dis. 97:231-244

Voglmayr, H., Castlebury, L. A., and Jaklitsch, W. M. 2017. Juglanconis gen. nov. on Juglandaceae, and the new family Juglanconidaceae (Diaporthales). Persoonia 38:136-155.

White, T. J., Bruns, T., Lee, S., and Taylor, J. 1990. Amplification and direct sequencing of fungi ribosomal RNA genes for phylogenetics. Pages 315-322 in: PCR Protocols: A Guide to Methods and Applications. M. A. Innis, D. H Gelfand, J. J. Sninsky, and T. J. White, eds. Academic Press, San Diego, CA.

Zhao, S. Z., Guo, K. F., He, L., and Yiming, A. 2018. First report of Cytospora nivea causing Cytospora canker on walnut (Juglans regia L.) in the Tianshan Mountains Region of Xinjiang, China. Plant Dis. 102:2640. 